\title{
Homoclinic Points in the Composition of Two Reflections
}

\author{
BY
}

\section{ERIK JENSEN}

A thesis submitted to the Graduate Program in Mathematics and Statistics in conformity with the requirements for the degree of Doctor of Philosophy

Queen's University

Kingston, Ontario, Canada

September, 2013

Copyright (C) Erik Jensen, 2013 


\section{Abstract}

We examine a class of planar area preserving mappings and give a geometric condition that guarantees the existence of homoclinic points.

To be more precise, let $f, g: \mathbb{R} \rightarrow \mathbb{R}$ be $C^{1}$ functions with domain all of $\mathbb{R}$. Let $F: \mathbb{R}^{2} \rightarrow \mathbb{R}^{2}$ denote a horizontal reflection in the curve $x=-f(y)$, and let $G: \mathbb{R}^{2} \rightarrow \mathbb{R}^{2}$ denote a vertical reflection in the curve $y=g(x)$. We consider maps of the form $T=G \circ F$ and show that a simple geometric condition on the fixed point sets of $F$ and $G$ leads to the existence of a homoclinic point for $T$. 


\section{Acknowledgements}

Thanks to

Dan Offin

Leo Jonker

Jennifer Read

my Family

and everyone else who has helped me along the way. 


\section{Statement of Originality}

What follows is original work by the author. All results from the work of others are referenced appropriately.

Erik Jensen

September, 2013 


\section{Contents}

$\begin{array}{ll}\text { Abstract } & \text { ii }\end{array}$

Acknowledgements $\quad$ iii

Statement of Originality iv

Table of Contents $\quad$ v

List of Figures $\quad$ vii

$\begin{array}{ll}\text { Chapter 1: Introduction } & 1\end{array}$

$\begin{array}{lr}\text { Chapter 2: Background Material } & 8\end{array}$

2.1 The Horseshoe, Topological Conjugacy, and Symbolic Dynamics . . . . . . . . . . 8

2.2 Stable and Unstable Manifolds, Homoclinic Points, and a Theorem of Smale . . . . . 14

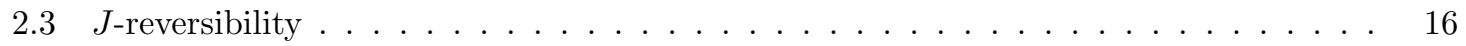

$\begin{array}{lr}\text { Chapter 3: Original Research } & 19\end{array}$

3.1 Preliminaries and Statement of Main Theorem _ . . . . . . . . . . . . . . . . . 19

3.2 Properties of Mappings $T:[f, g] \ldots \ldots \ldots \ldots \ldots \ldots \ldots \ldots \ldots$

3.3 A Closer Look at the Hyperbolic Fixed Points . . . . . . . . . . . . . . . . . . . . 29

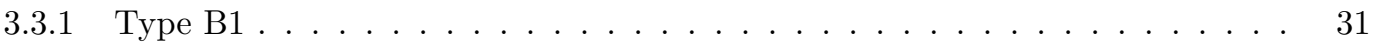

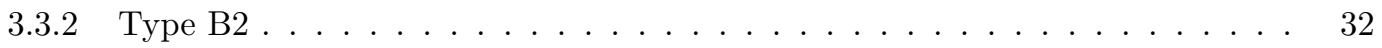

3.3 .3 Type $\mathrm{A} 1 \ldots \ldots \ldots \ldots \ldots \ldots \ldots \ldots$ 


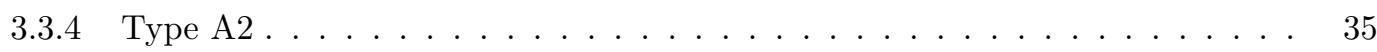

3.4 On the Existence of Heteroclinic Connections . . . . . . . . . . . . . . . . 36

3.5 On The Existence of Homoclinic Points _ . . . . . . . . . . . . . . . . . 38

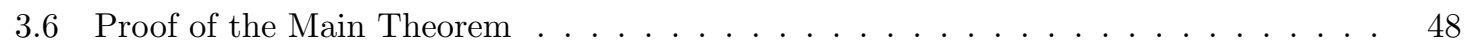

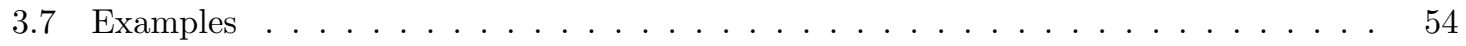

3.8 The Challenge of Proving Conjecture $1.0 .1 \ldots \ldots \ldots \ldots \ldots$

3.9 General Conditions that Guarantee the Existence of an Embedded Horseshoe . . . . 64

$\begin{array}{ll}\text { Chapter 4: Further Research } & 68\end{array}$

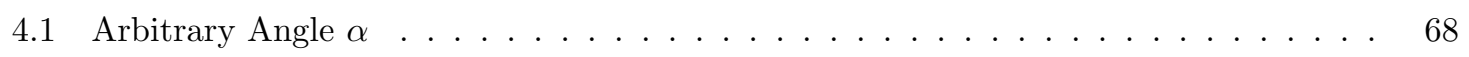

$\begin{array}{ll}\text { Bibliography } & 72\end{array}$ 


\section{List of Figures}

$1.1 H_{n}$ is a perpendicular reflection in $\mathcal{L}$ followed by a horizontal reflection in $\mathcal{P}_{n} \ldots \ldots$

1.2 The effect of $T:[f, g, \alpha]$ for $f(x)=(x+1)^{2}, g(x)=1$, and $\alpha=\frac{\pi}{4} . \ldots \ldots \ldots$

1.3 The effect of $T:[f, g]$ for $f(x)=x^{2}$ and $g(x)=x+2 \ldots \ldots \ldots \ldots$

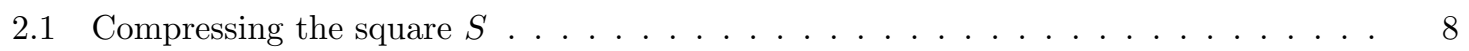

2.2 The resulting rectangle is stretched $\ldots \ldots \ldots \ldots \ldots \ldots$

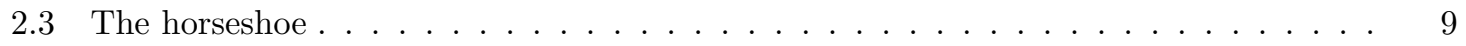

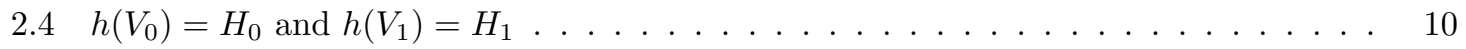

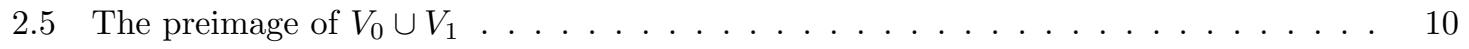

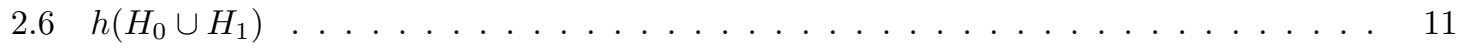

3.1 One iterate of a point $w$ under the map $T \ldots \ldots \ldots \ldots \ldots \ldots \ldots$

3.2 The lighter shaded region is above $\mathcal{G}$ and the darker shaded region shaded is below $\mathcal{G} . \quad 22$

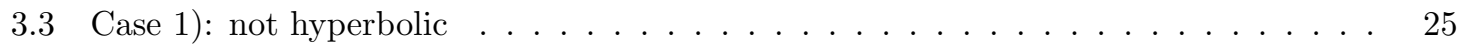

3.4 Case 2): the type B1 hyperbolic point $\ldots \ldots \ldots \ldots \ldots \ldots \ldots \ldots \ldots \ldots$

3.5 Case 3): the type B2 hyperbolic point $\ldots \ldots \ldots \ldots \ldots \ldots$

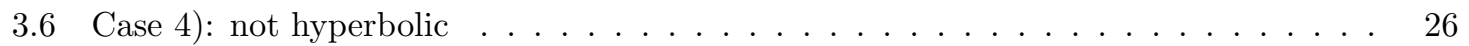

3.7 Case 5$)$ : the type A1 hyperbolic point $\ldots \ldots \ldots \ldots \ldots \ldots \ldots$

3.8 Case 6$)$ : the type $\mathrm{A} 2$ hyperbolic point $\ldots \ldots \ldots \ldots \ldots \ldots \ldots$

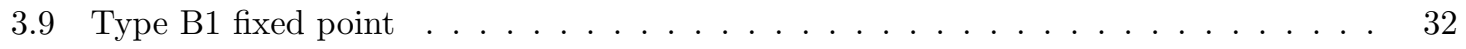

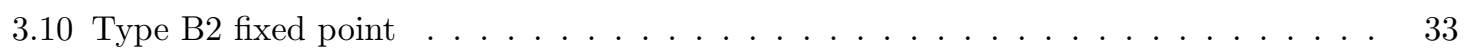




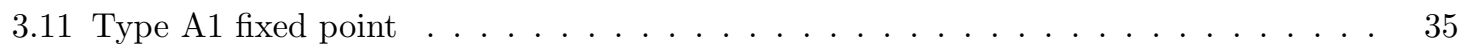

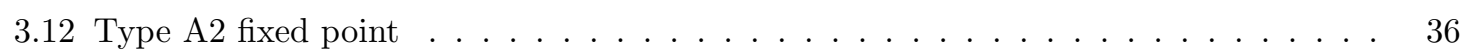

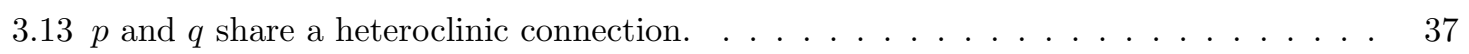

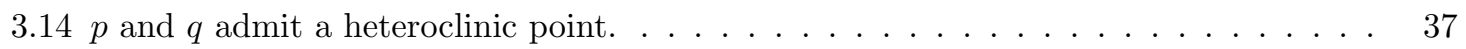

3.15 A heteroclinic connection between type A and type B fixed points contradicts the

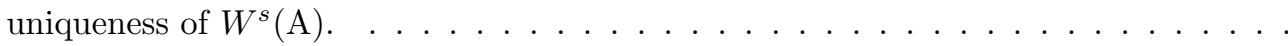

3.16 Cases 1, 2, 3, and 4. The red arrows indicate the initial slope of the unstable manifold of the fixed point, and the blue arrows indicate the initial slope of the stable manifold of the fixed point. . . . . . . . . . . . . . . . . .

3.17 Cases 5, 6, 7, and 8. The red arrows indicate the initial slope of the unstable manifold of the fixed point, and the blue arrows indicate the initial slope of the stable manifold

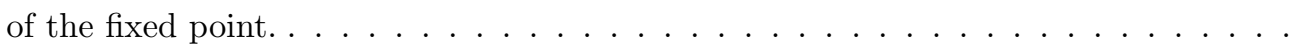

3.18 Four ways that $\mathcal{F}$ and $\mathcal{G}$ can intersect exactly once when one is linear and the other is quadratic. . . . . . . . . . . . . . . . . . . . . . .

3.19 If $\mathcal{F}$ and $\mathcal{G}$ are both quadratic, then the only way they can intersect exactly once is for the intersection point to be a tangency. . . . . . . . . . . . . .

3.20 Four ways that $\mathcal{F}$ and $\mathcal{G}$ can intersect exactly four times if they are both quadratic. Unstable manifolds are in red and stable manifolds are in blue. . . . . . . . . . .

3.21 Case 5: $T:[f, g]$ with $f(x)=\frac{3}{8} x^{2}-\frac{3}{2} x$ and $g(x)=x$. The blue and red curves are the stable and unstable manifolds, respectively, of the type A1 hyperbolic fixed point at $(0,0)$. The other fixed point is $\left(\frac{4}{3}, \frac{4}{3}\right)$ and is type B elliptic. . . . . . .

3.22 Case 6: $f(x)=x^{2}$ and $g(x)=x^{2}$. The blue and red curves are the stable and unstable manifolds, respectively, of the type A2 hyperbolic fixed point at $(-2,2)$. The other fixed point is $(0,0)$ and has a double -1 eigenvalue. $\ldots \ldots \ldots \ldots$ 
3.23 Case 7: $f(x)=2-x^{2}$ and $g(x)=x^{2}-4.25 x+2.5$. The blue and red curves are the stable and unstable manifolds, respectively, of the type A1 hyperbolic fixed point at $(4.25,2.5)$. The other fixed points are $(0.25,1.5)$ which is type B2 hyperbolic, and $(2,-2)$ which has a double 1 eigenvalue. . . . . . . . . . . . . .

3.24 Case 8: $f(x)=2-x^{2}$ and $g(x)=x^{2}-2$. The blue and red curves are the stable and unstable manifolds, respectively, of the type A2 hyperbolic fixed point at $(-1,-1)$. The other fixed points are $(2,2)$ which is type A1 hyperbolic, $\left(\frac{-1-\sqrt{5}}{2}, \frac{-1+\sqrt{5}}{2}\right)$ which is type B2 hyperbolic, and $\left(\frac{-1+\sqrt{5}}{2}, \frac{-1-\sqrt{5}}{2}\right)$ which is type B1 hyperbolic. . . . . . 58

$3.25 T:[f, g]$ with $f(x)=0.59 x(x-2)$ and $g(x)=x$. The blue and red curves are the stable and unstable manifolds, respectively, of the A1 fixed point at $(0,0) \ldots \ldots$.

3.26 Zooming in on the homoclinic loop for $T:[f, g]$ with $f(x)=0.59 x(x-2)$ and $g(x)=x$. The blue and red curves are the stable and unstable manifolds, respectively, of the

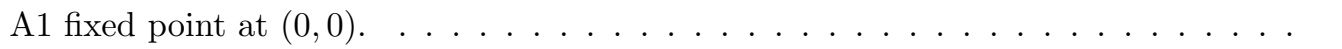

$3.27 T:[f, g]$ with $f(x)=0.59 x(x-2)$ and $g(x)=x$. The trajectories of points inside the homoclinic loop all seem to lie on invariant curves. . . . . . . . . . . . . . . . .

3.28 An extremely close up view of $W^{u}(0,0)$ (in red) and $W^{s}(0,0)$ (in blue) for $T:[f, g]$ with $f(x)=0.59 x(x-2)$ and $g(x)=x \ldots \ldots \ldots \ldots$

3.29 A tangential crossing at the homoclinic point $\chi \ldots \ldots \ldots 6$

4.1 The effect of $T:[f, g, \alpha]$ for $f(x)=(x+1)^{2}, g(x)=1$, and $\alpha=\frac{\pi}{4} . \ldots \ldots \ldots$ 


\section{Chapter 1}

\section{Introduction}

In 1960, Stephen Smale discovered the horseshoe. He gives an account of this discovery in his article 'Finding a horseshoe on the beaches of Rio' [S2]. The new science of chaotic dynamics was beginning to take shape, and Smale wanted to understand how a deterministic dynamical system could generate behaviour that appeared random. Using symbolic dynamics and his topology background, Smale considered the horseshoe mapping, and related the orbits to infinite sequences of coin flips. This was equivalent to showing that the horseshoe map is topologically conjugate to the shift map on the space of two sided infinite binary sequences.

The horseshoe map is quite important because it seems to be the 'prototypical' chaotic behaviour, in that most chaotic systems contain an embedded horseshoe. Using some results of Poincaré, Smale was able to make the above statement more precise. Poincaré, while studying the three body problem, discovered the homoclinic point, a point whose forward and backward orbits approach the same fixed point. An account of Poincaré's research can be found in June Barrow-Green's book 'Poincaré and the three body problem' [B-G]. Poincaré noted that the behaviour of a dynamical system near a homoclinic point became extremely complex. Smale, in his 1965 paper 'Diffeomorphisms with many periodic points' [S1], refined this idea and proved that if a homoclinic point for a dynamical system satisfied a certain condition(transversality), then the 
system contained an embedded horseshoe.

One of the first examples of a chaotic dynamical system was discovered by Edward Lorenz in 1963. He published his discovery in the Journal of Atmospheric Science, under the title 'Deterministic non-periodic flow' [L]. The system was defined by three coupled first order differential equations, and the solutions were curves in $\mathbb{R}^{3}$. This system was of great interest since all trajectories tended towards a set of measure zero with highly complex structure - a 'strange attractor'. However, numerical investigation of the Lorenz system proved difficult.

In 1976, the French astronomer M. Hénon published a paper titled 'A two dimensional mapping with a strange attractor' [H1]. In this paper, Hénon attempted to simplify the Lorenz system, so that it could be investigated more thoroughly. His approach was to look for a simple map that had the same essential properties as the Lorenz system. The result of Hénon's work was the map

$$
H\left(\begin{array}{l}
x \\
y
\end{array}\right)=\left(\begin{array}{c}
1+y-a x^{2} \\
b x
\end{array}\right)
$$

which has become known as the Hénon map.

In their 1979 paper 'Shift automorphisms in the Hénon mapping' [DN], R. Devaney and Z. Nitecki investigated the behaviour of the Hénon map for various parameter values. They defined a square $S$, centered at the origin, with sidelength a function of the parameters $a$ and $b$. Then, they proved that for $a \geq 2(1+|b|)^{2}$, the set

$$
\bigcap_{n \in \mathbb{Z}} H^{n}(S)
$$

is a topological horseshoe. Their proof did not make use of Smale's result that a transverse homoclinic point implies the existence of a horseshoe. We note that in the measure-preserving case, Devaney and Nitecki's result proves that $H$ has a horseshoe for $a \geq 8$.

In 1984, Devaney published a paper titled 'Homoclinic bifurcations and the area-conserving Hénon mapping' [D1], in which he defined the important concept of $J$-reversibility. Devaney showed that if an area-preserving map is $J$-reversible, then there is a convenient way to prove the 
existence of a homoclinic point for that map. He then used this result to prove that the Hénon map contained a homoclinic point for $a>-1$, provided that $a \neq 0$. However, he made no assertions about the transversality of this homoclinic point.

In 1995, Ray Brown continued the investigation of the measure-preserving Hénon map in his paper 'Horseshoes in the measure-preserving Hénon map' [Br]. He used Devaney's concept of $J$-reversibility and some geometric methods to show that $H$ had a homoclinic point for $a>0$. Brown's geometric methods allowed him to obtain some facts about the homoclinic point, and he used these facts in a contradiction argument to show that the homoclinic point had to be transverse. Thus, Brown proved that the measure-preserving Hénon map contains a horseshoe for $a>0$. This result improved upon Devaney and Nitecki's earlier result that $H$ contains a horseshoe for $a \geq 8$.

In the author's M.Sc. thesis [J1], Brown's proof is studied quite closely, and his methods are adapted to investigate the generalized even Hénon maps, which are given by

$$
H_{n}\left(\begin{array}{l}
x \\
y
\end{array}\right)=\left(\begin{array}{c}
1+y-a x^{2 n} \\
-x
\end{array}\right)
$$

The author obtained the result that $H_{n}$ contains a horseshoe for $a>A_{n}$, where $A_{n}<\frac{1}{n}$ for all $n$.

While pursuing this problem, it became clear that all the maps $H_{n}$ had a common geometric structure. First, each is the composition of two reflections: $H_{n}=P_{n} \circ L$, where $L$ is a perpendicular reflection in a line $\mathcal{L}$ and $P_{n}$ is a horizontal reflection in a curve $\mathcal{P}_{n}$ of degree $2 n$ (see Figure 1.1). Second, the curves $\mathcal{L}$ and $\mathcal{P}_{n}$ cross more than once, and third, $\mathcal{P}_{n}$ will have either a global maximum or a global minimum. It seems that these three properties together lead to chaotic behaviour.

In this thesis we generalize the maps $H_{n}$ in order to investigate the role of this common geometric structure.

The key facts are that the maps are the composition of two reflections, where the reflections are through graphs of functions, and that these graphs are oriented at an angle to each other. So the 


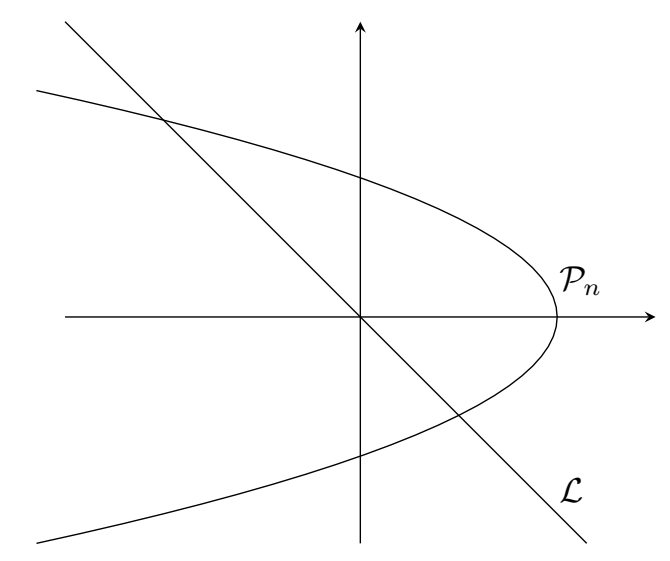

Figure 1.1: $H_{n}$ is a perpendicular reflection in $\mathcal{L}$ followed by a horizontal reflection in $\mathcal{P}_{n}$.

key elements are two graphs and an angle. Take these and construct a family of maps as follows. Take two functions $f$ and $g$ and draw their graphs. Rotate the graph of $f$ by an angle $\alpha$. Then let $F$ denote the reflection through the rotated graph of $f$ in the direction $(-\sin \alpha, \cos \alpha)$. Let $G$ denote the vertical reflection through the graph of $g$. Then consider the composition $G \circ F$ of the two reflections. We let $\mathcal{F}$ denote the fixed point set of $F$ (so that $\mathcal{F}$ is the rotated graph of $f$ ) and let $\mathcal{G}$ denote the fixed point set of $G$ (so that $\mathcal{G}$ is the graph of $g$ ).

So, two functions $f$ and $g$ and an angle $\alpha$ define a map. We write this as $T:[f, g, \alpha]$. For example, Figure 1.2 illustrates the map $T:[f, g, \alpha]$ where $f(x)=(x+1)^{2}, g(x)=1$, and $\alpha=\frac{\pi}{4}$.

As one begins investigating this problem, it quickly becomes clear that letting $\alpha$ be arbitrary caused significant technical difficulties. By fixing the angle, we can still study the essential nature of the problem while keeping computational headaches to a minimum. In this thesis, we fix $\alpha=\frac{\pi}{2}$ and suppress the dependence on $\alpha$ in the notation. So $T:[f, g]$ could be written as $T:\left[f, g, \frac{\pi}{2}\right]$, but since $\alpha$ is always $\frac{\pi}{2}$ for this thesis we just write it as $T:[f, g]$. Note that $T:[f, g]$ is a horizontal reflection in the curve $x=-f(y)$ followed by a vertical reflection in the curve $y=g(x)$. For example, Figure 1.3 illustrates the map $T:[f, g]$ where $f(x)=x^{2}$ and $g(x)=x+2$.

Once the maps $T:[f, g]$ have been defined (a more thorough definition is given in Section 3.1), it is natural to become intrigued by the idea that two simple transformations could be combined 


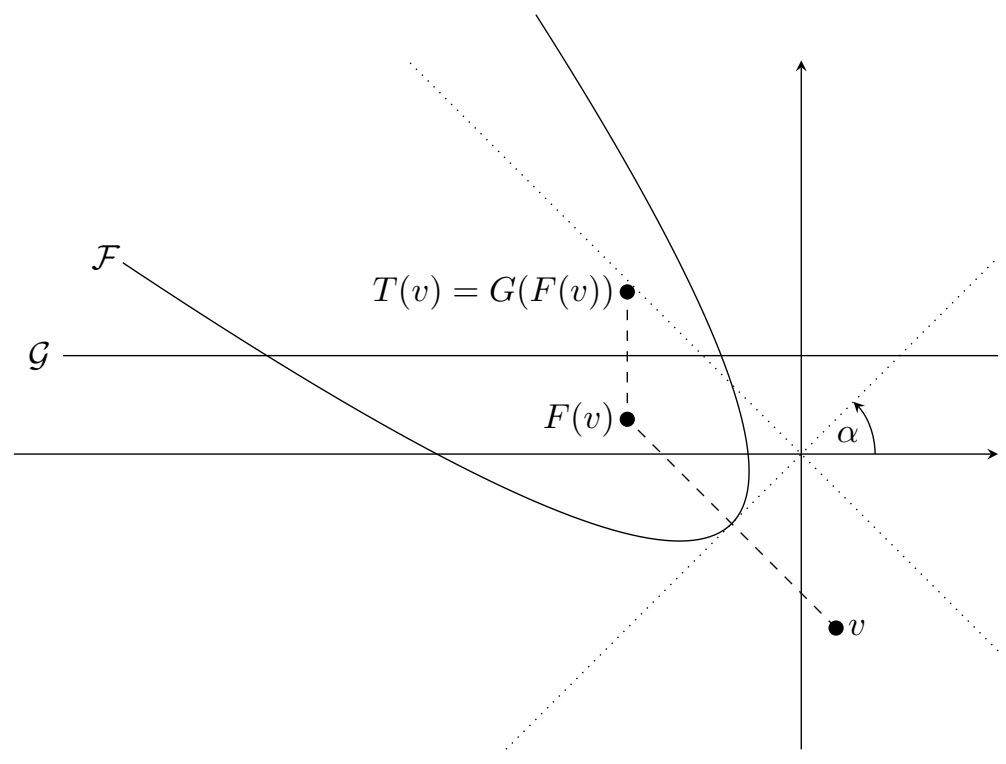

Figure 1.2: The effect of $T:[f, g, \alpha]$ for $f(x)=(x+1)^{2}, g(x)=1$, and $\alpha=\frac{\pi}{4}$.

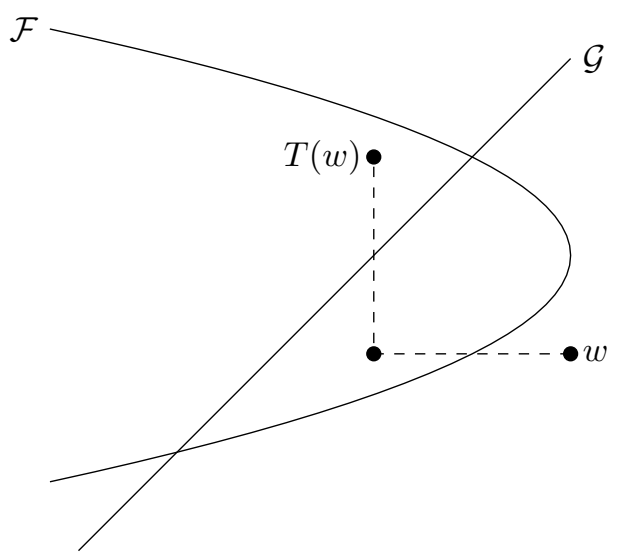

Figure 1.3: The effect of $T:[f, g]$ for $f(x)=x^{2}$ and $g(x)=x+2$.

together to produce complex behaviour. The following question presents itself: just how simple could the functions $f$ and $g$ be such that the map $T:[f, g]$ would still be chaotic? Informally, the main result of this thesis is that $f$ and $g$ can be extremely simple functions (linear or quadratic) and the associated map $T:[f, g]$ will contain a homoclinic point if $\mathcal{F}$ and $\mathcal{G}$ cross more than once (recall that $\mathcal{F}$ denotes the graph of $x=-f(y)$ and $\mathcal{G}$ denotes the graph of $y=g(x)$ ). A formal 
statement of the Main Theorem reads as follows.

Main Theorem. Let $f, g: \mathbb{R} \rightarrow \mathbb{R}$ be $C^{1}$ functions with domain all of $\mathbb{R}$, and consider the associated mapping $T:[f, g]$. Let $\mathcal{F}$ denote the graph of $x=-f(y)$ and $\mathcal{G}$ denote the graph of $y=g(x)$, and assume that all intersections of $\mathcal{F}$ and $\mathcal{G}$ are transverse. Then the following two statements are true.

1. If one of $f$ or $g$ is quadratic and the other is linear, then the map $T$ will admit a homoclinic point if and only if $\mathcal{F}$ and $\mathcal{G}$ intersect more than once.

2. If $f$ and $g$ are both quadratic, then the map $T$ will admit a homoclinic point if and only if $\mathcal{F}$ and $\mathcal{G}$ intersect more than once.

A natural question to consider is the following. Given the same hypothesis as the Main Theorem, can we prove the stronger conclusion that $T:[f, g]$ admits an embedded horseshoe? In other words, can we prove the following conjecture?

Conjecture 1.0.1. Let $f, g: \mathbb{R} \rightarrow \mathbb{R}$ be $C^{1}$ functions with domain all of $\mathbb{R}$, and consider the associated mapping $T:[f, g]$. Let $\mathcal{F}$ denote the graph of $x=-f(y)$ and $\mathcal{G}$ denote the graph of $y=g(x)$, and assume that all intersections of $\mathcal{F}$ and $\mathcal{G}$ are transverse. Then the following two statements are true.

1. If one of $f$ or $g$ is quadratic and the other is linear, then the map $T$ will admit an embedded horseshoe if and only if $\mathcal{F}$ and $\mathcal{G}$ intersect more than once.

2. If $f$ and $g$ are both quadratic, then the map $T$ will admit an embedded horseshoe if and only if $\mathcal{F}$ and $\mathcal{G}$ intersect more than once.

Conjecture 1.0.1 may well be true, but proving it promises to be extremely difficult. In section 3.8 we will look at an example that illustrates how delicate the situation is. It could be that guaranteeing the existence of a horseshoe requires further conditions on the class of maps $T:[f, g]$. We investigate one possibe extra condition in Section 3.9. 
This thesis is structured as follows. In Chapter 2 we define the horseshoe and introduce important results from Smale and Devaney. Chapter 3 begins by giving a precise statement of the problem we are considering and then builds up to Section 3.6, in which we prove the Main Theorem. In Section 3.7, we look at several concrete examples, and in Section 3.8 we discuss the difficulty of proving Conjecture 1.0.1. Section 3.9 gives some general conditions that guarantee the existence of a horseshoe for maps $T:[f, g]$. In Chapter 4 we reintroduce the arbitrary angle $\alpha$. 


\section{Chapter 2}

\section{Background Material}

\subsection{The Horseshoe, Topological Conjugacy, and Symbolic Dynamics}

In this section, we define the horseshoe map, and show that it is topologically conjugate to a simple map on the space of two-sided infinite sequences.

Let $S$ be a square in $\mathbb{R}^{2}$. The horseshoe map $h: S \rightarrow \mathbb{R}^{2}$ is continuous, one to one, but not onto. $h$ acts on the square $S$ as follows. First, $h$ linearly contracts $S$ in the vertical direction by a factor of $\delta<\frac{1}{2}$. This is shown in Figure 2.1.

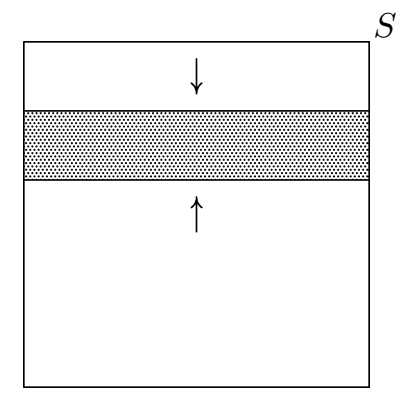

Figure 2.1: Compressing the square $S$ 
Then, $h$ linearly expands the resulting rectangle in the horizontal direction by a factor of $\frac{1}{\delta}$.

This is shown in Figure 2.2.

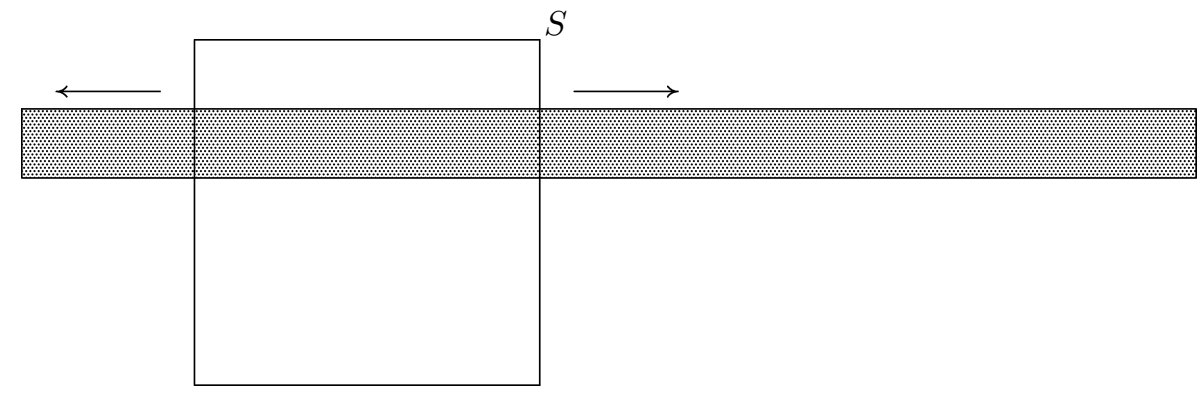

Figure 2.2: The resulting rectangle is stretched

Finally, $h$ folds the long, thin rectangle into a horseshoe shape and puts it back inside $S$. Figure 2.3 shows the result of this transformation.

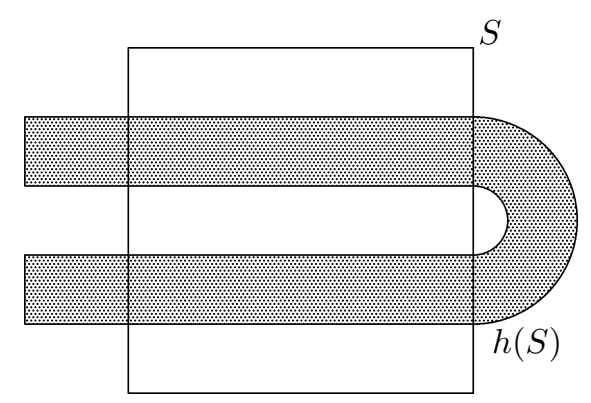

Figure 2.3: The horseshoe

We want to describe the set

$$
\Lambda=\left\{\boldsymbol{q} \in S \mid h^{k}(\boldsymbol{q}) \in S \quad \text { for all } k \in \mathbb{Z}\right\} .
$$

This is the set of points whose orbits under iteration of $h$ lie in $S$ for all time.

We note that the preimage of $S$ consists of two vertical rectangles $V_{0}$ and $V_{1}$, which are mapped to two horizontal rectangles $H_{0}$ and $H_{1} . V_{0}$ and $V_{1}$ have width $\delta$, and $H_{0}$ and $H_{1}$ have height $\delta$. $H_{0}, H_{1}, V_{0}$, and $V_{1}$ are shown in Figure 2.4. 

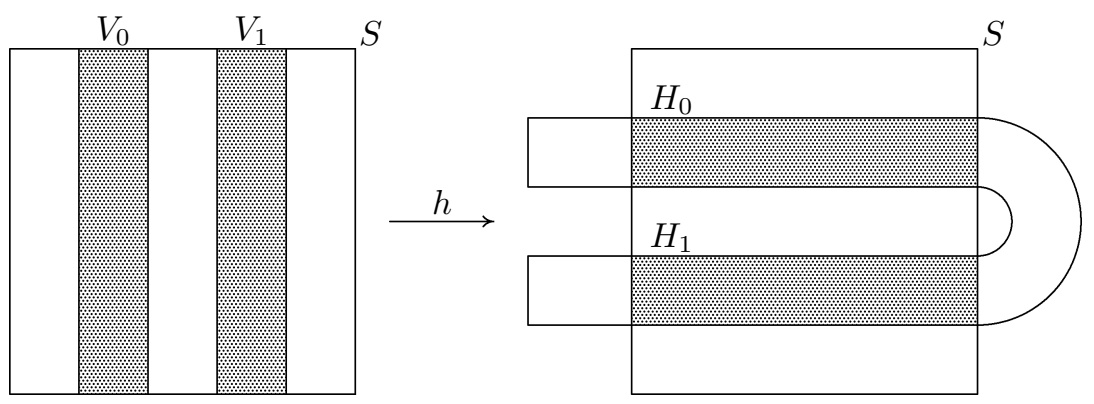

Figure 2.4: $h\left(V_{0}\right)=H_{0}$ and $h\left(V_{1}\right)=H_{1}$

We also note that if $\boldsymbol{q} \notin V_{0} \cup V_{1}$, then $h(\boldsymbol{q}) \notin S$. So, any point that is not in either $V_{0}$ or $V_{1}$ is mapped outside of $S$.

The preimage of $V_{0}$ and $V_{1}$ is a pair of smaller rectangles inside of $V_{0}$ and $V_{1}$. Figure 2.5 illustrates this.
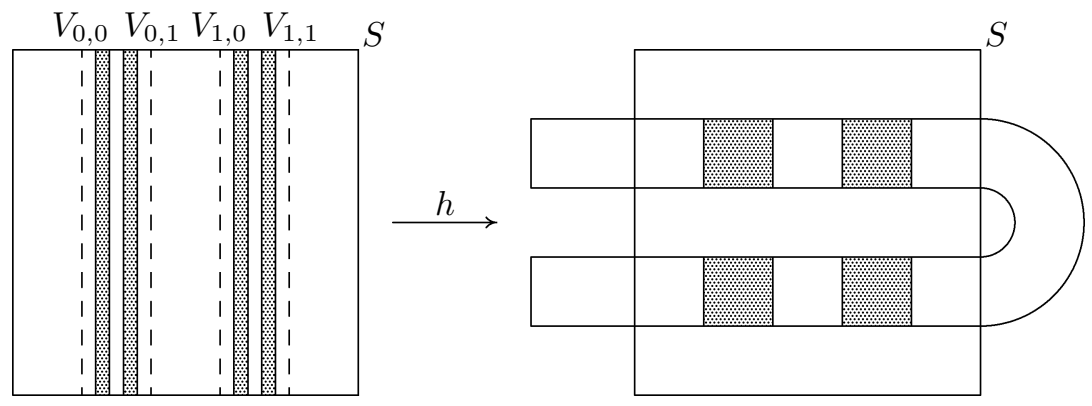

Figure 2.5: The preimage of $V_{0} \cup V_{1}$

Similarly, the preimage of $V_{0,0}, V_{0,1}, V_{1,0}$, and $V_{1,1}$ is a set of eight vertical rectangles, two inside each of the $V_{i, j}$.

We make the following definition

$$
\Lambda_{+}=\left\{\boldsymbol{q} \in S \mid h^{k}(\boldsymbol{q}) \in S \quad \text { for all } k \in \mathbb{N}\right\}
$$


We observe that

$$
\begin{aligned}
& h^{0}(\boldsymbol{q}) \in S \Rightarrow \boldsymbol{q} \in S \\
& h^{1}(\boldsymbol{q}) \in S \Rightarrow \boldsymbol{q} \in V_{0} \cup V_{1} \\
& h^{2}(\boldsymbol{q}) \in S \Rightarrow \boldsymbol{q} \in V_{0,0} \cup V_{0,1} \cup V_{1,0} \cup V_{1,1}
\end{aligned}
$$

and we conclude that $\Lambda_{+}$is a Cantor set of vertical line segments.

Now we define the set

$$
\Lambda_{-}=\left\{\boldsymbol{q} \in S \mid h^{-k}(\boldsymbol{q}) \in S \quad \text { for all } k \in \mathbb{N}\right\}
$$

We observe that

$$
h^{-1}(\boldsymbol{q}) \in S \Rightarrow \boldsymbol{q} \in H_{0} \cup H_{1} .
$$

Also, $h$ maps $H_{0}$ and $H_{1}$ as in Figure 2.6.

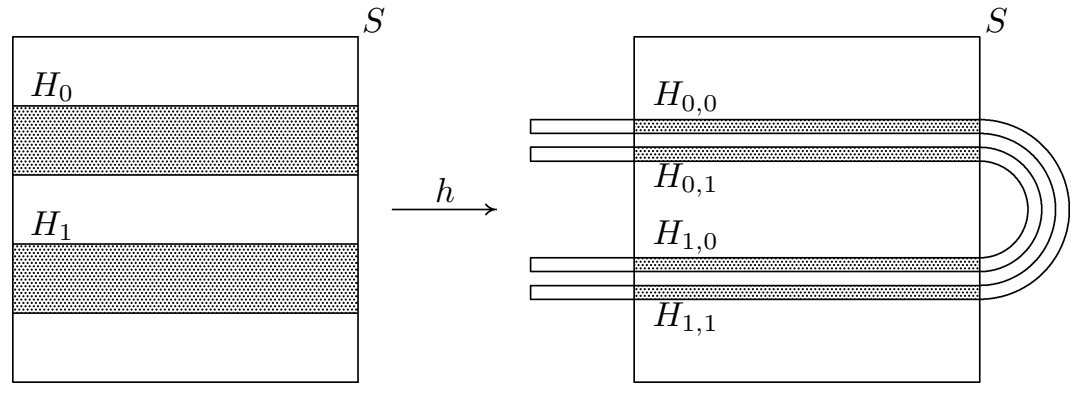

Figure 2.6: $h\left(H_{0} \cup H_{1}\right)$

So we see that

$$
h^{-2}(\boldsymbol{q}) \in S \Rightarrow \boldsymbol{q} \in H_{0,0} \cup H_{0,1} \cup H_{1,0} \cup H_{1,1}
$$

and we conclude that $\Lambda$ - is a Cantor set of horizontal line segments.

It is clear that $\Lambda=\Lambda_{+} \cap \Lambda_{-}$

Now we may make the transition to symbolic dynamics. Let $\Sigma_{2}$ denote the set of all doubly infinite binary sequences, so that

$$
\Sigma_{2}=\left\{\left(\ldots s_{-2} s_{-1} . s_{0} s_{1} s_{2} \ldots\right) \mid s_{i} \text { is either } 0 \text { or } 1\right\}
$$


We define a metric

$$
d\left[\left(\ldots s_{-1} \cdot s_{0} \ldots\right),\left(\ldots t_{-1} \cdot t_{0} \ldots\right)\right]=\sum_{i=-\infty}^{\infty} \frac{\left|s_{i}-t_{i}\right|}{2^{|i|}}
$$

so that $\left(\Sigma_{2}, d\right)$ is a metric space.

Now, for each point $\boldsymbol{p} \in \Lambda$, we define a doubly infinite sequence

$$
S(\boldsymbol{p})=\left(\ldots s_{-2} s_{-1} . s_{0} s_{1} s_{2} \ldots\right)
$$

by the rules:

1. If $h^{i}(\boldsymbol{p}) \in V_{0}$, then set $s_{i}=0$.

2. If $h^{i}(\boldsymbol{p}) \in V_{1}$, then set $s_{i}=1$.

We note that $S$ is a homeomorphism from $\Lambda$ to $\Sigma_{2}$.

We also note that a half sequence $\left(. s_{0} s_{1} s_{2} \ldots\right)$ specifies a vertical line segment in $\Lambda_{+}$. Similarly, a half sequence $\left(\ldots s_{-2} s_{-1}\right.$.) specifies a horizontal line segment in $\Lambda_{-}$. So, a doubly infinite sequence $\left(\ldots s_{-2} s_{-1} . s_{0} s_{1} s_{2} \ldots\right)$ specifies both a horizontal and a vertical line segment and thus it specifies a unique point $\boldsymbol{p} \in \Lambda$.

Now, we define the shift map $\sigma: \Sigma_{2} \rightarrow \Sigma_{2}$ by

$$
\sigma\left(\ldots s_{-2} s_{-1} . s_{0} s_{1} s_{2} \ldots\right)=\left(\ldots s_{-2} s_{-1} s_{0} . s_{1} s_{2} \ldots\right)
$$

So the shift map shifts each sequence in $\Sigma_{2}$ one unit to the left. $\sigma$ is a homeomorphism.

We recall the definition of topological conjugacy.

Definition 2.1.1. Two maps $f$ and $g$ are topologically conjugate if there exists a homeomorphism $l$ such that $l \circ f=g \circ l$.

Now, we prove that $\left.h\right|_{\Lambda}$ is conjugate to $\sigma$ via $S$.

Proposition 2.1.1. $S: \Lambda \rightarrow \Sigma_{2}$ gives a topological conjugacy between $\sigma$ and $h$. 
Proof. We need to show that $S \circ h=\sigma \circ S$. Let $\boldsymbol{p} \in \Lambda$, with

$$
S(\boldsymbol{p})=\left(\ldots s_{-2} s_{-1} \cdot s_{0} s_{1} s_{2} \ldots\right)
$$

We note that $h(\boldsymbol{p}) \in \Lambda$ by definition. We have that

$$
S(h(\boldsymbol{p}))=\left(\ldots t_{-2} t_{-1} \cdot t_{0} t_{1} t_{2} \ldots\right)
$$

where

$$
t_{i}=k \Longleftrightarrow h^{i}(h(\boldsymbol{p})) \in V_{k} \Longleftrightarrow h^{i+1}(\boldsymbol{p}) \in V_{k}
$$

and

$$
\sigma(S(\boldsymbol{p}))=\left(\ldots s_{-2} s_{-1} s_{0} . s_{1} s_{2} \ldots\right)
$$

where

$$
s_{i}=k \Longleftrightarrow h^{i}(\boldsymbol{p}) \in V_{k} .
$$

So $t_{i-1}=s_{i}$ for all $i \in \mathbb{Z}$. Thus $S \circ h=\sigma \circ S$.

In this proposal, the phrase 'contains an embedded horseshoe' is used frequently. The following definition explains precisely what this means.

Definition 2.1.2. A map $T: \mathbb{R}^{2} \rightarrow \mathbb{R}^{2}$ is said to contain an embedded horseshoe if $T$ has an invariant set $\Theta$ such that $\left.T\right|_{\Theta}$ is topologically conjugate to $\left.h\right|_{\Lambda}$. 


\subsection{Stable and Unstable Manifolds, Homoclinic Points, and a Theorem of Smale}

In this section we present some basic definitions that are used frequently throughout this thesis.

Let $T: \mathbb{R}^{2} \rightarrow \mathbb{R}^{2}$ be a $C^{2}$ invertible map. Let $\boldsymbol{v}$ be a fixed point of $T$.

Definition 2.2.1. The unstable manifold of $T$ at the point $\boldsymbol{v}$ is the set

$$
\left\{\boldsymbol{z} \mid T^{-n}(\boldsymbol{z}) \rightarrow \boldsymbol{v}\right\}
$$

and is denoted by $W^{u}(\boldsymbol{v})$. The stable manifold of $T$ at the point $\boldsymbol{v}$ is the set

$$
\left\{\boldsymbol{z} \mid T^{n}(\boldsymbol{z}) \rightarrow \boldsymbol{v}\right\}
$$

and is denoted by $W^{s}(\boldsymbol{v})$.

Definition 2.2.2. A fixed point $\boldsymbol{v}$ of $\mathrm{T}$ is hyperbolic if $\mathrm{D} T(\boldsymbol{v})$ has no eigenvalues on the unit circle, where $\mathrm{D} T(\boldsymbol{v})$ is the Jacobian matrix at $\boldsymbol{v}$.

Theorem 2.2.1 (Stable and Unstable Manifold Theorem). Let $\boldsymbol{v}$ be a hyperbolic fixed point for T. Then $W^{u}(\boldsymbol{v})$ and $W^{s}(\boldsymbol{v})$ are smooth curves in $\mathbb{R}^{2}$.

A proof of this theorem can be found in Devaney's book 'An Introduction to Chaotic Dynamical Systems' [D2]. The Stable and Unstable Manifold Theorem is very important for this thesis, since many of the results in other sections depend implicitly on the fact that the unstable manifold of a hyperbolic fixed point is continuous.

Definition 2.2.3. A homoclinic point of $T$ is a point $\chi \neq \boldsymbol{v}$ in the intersection of $W^{u}(\boldsymbol{v})$ and $W^{s}(\boldsymbol{v})$.

Definition 2.2.4. A homoclinic point $\chi$ is called transverse if the direction of $W^{u}(\boldsymbol{v})$ at $\chi$ and the direction of $W^{s}(\boldsymbol{v})$ at $\chi$ are not collinear. A homoclinic point $\chi$ is called degenerate or nontransverse if the directions of $W^{u}(\boldsymbol{v})$ and $W^{s}(\boldsymbol{v})$ are collinear at $\chi$. 
Transverse homoclinic points of $T$ have important consequences for the dynamics of $T$.

Theorem 2.2.2 (Smale). If $T$ has a transverse homoclinic point, then $T$ has an embedded horseshoe.

A proof of Smale's theorem can be found in his paper 'Diffeomorphisms with many periodic points' [S1].

In the next section, we look at the problem of finding a homoclinic point(transverse or not) for a map $T$. 


\section{$2.3 \quad J$-reversibility}

The results of this section are taken from Devaney's paper 'Homoclinic bifurcations and the area-conserving Hénon mapping' [D1].

In the previous section, we saw that if a map $T$ has a transverse homoclinic point, then $T$ has a horseshoe. In this section, we look at the problem of finding a homoclinic point for a given map. The key idea is $J$-reversibility. If a map $T$ is $J$-reversible, then the problem of finding a homoclinic point for $T$ becomes much simpler.

A diffeomorphism $T: \mathbb{R}^{2 n} \rightarrow \mathbb{R}^{2 n}$ is called $J$-reversible if $T$ is topologically conjugate to $T^{-1}$ via a smooth diffeomorphism $J$, and $J$ satisfies certain conditions. Let fix $(J)$ denote the set of points in $\mathbb{R}^{2 n}$ that are fixed by $J$. The main result of this section states that if a diffeomorphism $T: \mathbb{R}^{2 n} \rightarrow \mathbb{R}^{2 n}$ is $J$-reversible and $\boldsymbol{p}$ is a saddle fixed point for $T$ with $\boldsymbol{p} \in \operatorname{fix}(J)$, then any $\boldsymbol{u} \in W^{u}(\boldsymbol{p}) \cap \operatorname{fix}(J)$ is a homoclinic point for $T$. This means that to find a homoclinic point for $T$, instead of looking for intersections of $W^{u}(\boldsymbol{p})$ and $W^{s}(\boldsymbol{p})$, one looks for intersections of $W^{u}(\boldsymbol{p})$ and $\operatorname{fix}(J)$. Since $\operatorname{fix}(J)$ is a far simpler set than $W^{s}(\boldsymbol{p})$, this result makes finding a homoclinic point for $T$ much easier. It is important to note that a homoclinic point found using this method may be transverse or nontransverse; this result says nothing about the nature of the homoclinic point found.

Now, we look at the details.

Let $T: \mathbb{R}^{2 n} \rightarrow \mathbb{R}^{2 n}$ be a diffeomorphism, and denote the identity map by $I$.

Definition 2.3.1. $J$ is called a reversible involution if $J$ is a smooth diffeomorphism of $\mathbb{R}^{2 n}$ that satisfies the following conditions:

1. $J \circ J=I$, ie. $J$ is an involution.

2. The dimension of $\operatorname{fix}(J)$ is $n$.

Definition 2.3.2. $T$ is called $J$-reversible if the following conditions hold: 
1. $J \circ T=T^{-1} \circ J$, ie. $T$ is topologically conjugate to $T^{-1}$ via $J$.

2. $J$ is a reversible involution.

For the rest of this section, we let $J$ be a reversible involution. In the following proposition, we state an interesting property of $J$-reversible diffeomorphisms.

Proposition 2.3.1. Let $T$ be $J$-reversible. Then $T$ can be written as the composition of two involutions.

Proof. We note that $T=(T \circ J) \circ J$, and that

$$
(T \circ J)^{2}=T \circ(J \circ T) \circ J=T \circ T^{-1} \circ J \circ J=I .
$$

So, both $T \circ J$ and $J$ are involutions, and we may write $T$ as the composition of two involutions.

We also note that if a diffeomorphism $G: \mathbb{R}^{2 n} \rightarrow \mathbb{R}^{2 n}$ is the composition of two reversible involutions $S_{1}$ and $S_{2}$, then $G$ is both $S_{1}$-reversible and $S_{2}$-reversible, since $S_{1} \circ\left(S_{1} \circ S_{2}\right)=\left(S_{2} \circ S_{1}\right) \circ S_{1}$ and $\left(S_{1} \circ S_{2}\right) \circ S_{2}=S_{2} \circ\left(S_{2} \circ S_{1}\right)$.

The following proposition will be necessary to prove the main result of this section.

Proposition 2.3.2. Let $T$ be $J$-reversible. Let $\boldsymbol{p}$ be a saddle fixed point for $T$ with $\boldsymbol{p} \in$ fix $(J)$. Let $W^{u}(\boldsymbol{p})$ and $W^{s}(\boldsymbol{p})$ denote the unstable and stable manifolds of $\boldsymbol{p}$, repectively. Then $J\left(W^{u}(\boldsymbol{p})\right)=W^{s}(\boldsymbol{p})$ and $J\left(W^{s}(\boldsymbol{p})\right)=W^{u}(\boldsymbol{p})$.

Proof. Let $\boldsymbol{x} \in W^{u}(\boldsymbol{p})$. This means that $\lim _{n \rightarrow \infty} T^{-n}(\boldsymbol{x})=\boldsymbol{p}$. Now we have

$$
\begin{aligned}
\boldsymbol{p}=J(\boldsymbol{p}) & =J\left(\lim _{n \rightarrow \infty} T^{-n}(\boldsymbol{x})\right) \\
& =\lim _{n \rightarrow \infty} J T^{-n}(\boldsymbol{x}) \\
& =\lim _{n \rightarrow \infty} T^{n} J(\boldsymbol{x}), \quad \text { since } J T^{-1}=T J . \\
& =\lim _{n \rightarrow \infty} T^{n}(J(\boldsymbol{x})) .
\end{aligned}
$$


So, we have that $J(\boldsymbol{x}) \in W^{s}(\boldsymbol{p})$ by definition. So far we have shown that $J W^{u}(\boldsymbol{p}) \subseteq W^{s}(\boldsymbol{p})$. Now, let $\boldsymbol{y} \in W^{s}(\boldsymbol{p})$, so that $\lim _{n \rightarrow \infty} T^{n}(\boldsymbol{y})=\boldsymbol{p}$. We have

$$
\begin{aligned}
\boldsymbol{p}=J(\boldsymbol{p}) & =J\left(\lim _{n \rightarrow \infty} T^{n}(\boldsymbol{y})\right) \\
& =\lim _{n \rightarrow \infty} J T^{n}(\boldsymbol{y}) \\
& =\lim _{n \rightarrow \infty} T^{-n}(J(\boldsymbol{y}))
\end{aligned}
$$

so that $J(\boldsymbol{y}) \in W^{u}(\boldsymbol{p})$. This implies that $\boldsymbol{y} \in J W^{u}(\boldsymbol{p})$ and thus we have $W^{s}(\boldsymbol{p}) \subseteq J W^{u}(\boldsymbol{p})$. So, we have proved that $J W^{u}(\boldsymbol{p})=W^{s}(\boldsymbol{p})$ and thus that $W^{u}(\boldsymbol{p})=J W^{s}(\boldsymbol{p})$ as well.

Now we prove the main result.

Theorem 2.3.1. Let $T$ be $J$-reversible. Let $\boldsymbol{p}$ be a hyperbolic fixed point for $T$ with $\boldsymbol{p} \in$ fix $(J)$. Let $W^{u}(\boldsymbol{p})$ and $W^{s}(\boldsymbol{p})$ denote the unstable and stable manifolds, respectively. Let $\boldsymbol{u} \in W^{u}(\boldsymbol{p}) \cap \operatorname{fix}(J)$, with $\boldsymbol{u} \neq \boldsymbol{p}$. Then $\boldsymbol{u}$ is a homoclinic point.

Proof. We have that $\boldsymbol{u} \in W^{u}(\boldsymbol{p})$ and $\boldsymbol{u} \in \operatorname{fix}(J)$. So, $\boldsymbol{u}=J(\boldsymbol{u}) \in J W^{u}(\boldsymbol{p})=W^{s}(\boldsymbol{p})$, using the previous proposition. So, $\boldsymbol{u} \in W^{u}(\boldsymbol{p}) \cap W^{s}(\boldsymbol{p})$ and since $\boldsymbol{u} \neq \boldsymbol{p}$ then $\boldsymbol{u}$ is a homoclinic point. 


\section{Chapter 3}

\section{Original Research}

\subsection{Preliminaries and Statement of Main Theorem}

We will consider planar maps that are the composition of two reflections. More specifically, let $f, g: \mathbb{R} \rightarrow \mathbb{R}$ be two functions. We consider mappings $T$ which are given by a horizontal reflection in the curve $x=-f(y)$ followed by a vertical reflection in the curve $y=g(x)$.

For example, if $f(t)=t^{2}$ and $g(t)=t+2$, then the map $T$ would be a horizontal reflection in the curve $x=-y^{2}$ followed by a vertical reflection in the curve $y=x+2$. This is illustrated in Figure 3.1.

To make this precise, we make the following definitions:

Let $f, g: \mathbb{R} \rightarrow \mathbb{R}$ be $C^{1}$ functions with domain all of $\mathbb{R}$.

Let $F: \mathbb{R}^{2} \rightarrow \mathbb{R}^{2}$ denote a horizontal reflection in the graph of $x=-f(y)$, and let $\mathcal{F}$ denote the fixed point set of $F$.

Let $G: \mathbb{R}^{2} \rightarrow \mathbb{R}^{2}$ denote a vertical reflection in the graph of $y=g(x)$, and let $\mathcal{G}$ denote the fixed point set of $G$.

The maps we consider are of the form $T=G \circ F$.

We note that $T$ is both $F$ and $G$ reversible (in the terminology of Section 2.3), since 


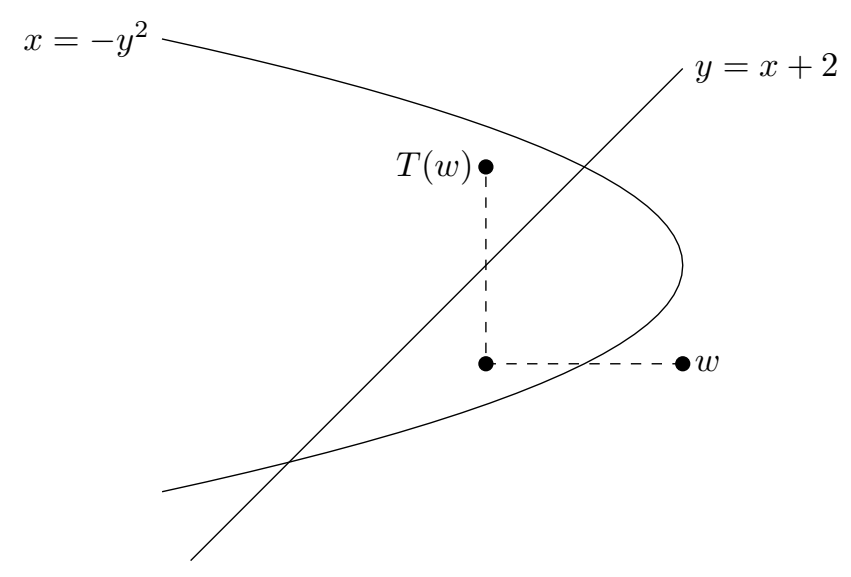

Figure 3.1: One iterate of a point $w$ under the map $T$.

$T \circ F=F \circ T^{-1}$ and $G \circ T=T^{-1} \circ G$.

The expressions $F, G$, and $T$ are given by

$$
\begin{aligned}
F\left(\begin{array}{l}
x \\
y
\end{array}\right) & =\left(\begin{array}{c}
-2 f(y)-x \\
y
\end{array}\right) \\
G\left(\begin{array}{l}
x \\
y
\end{array}\right) & =\left(\begin{array}{c}
x \\
2 g(x)-y
\end{array}\right) \\
T\left(\begin{array}{l}
x \\
y
\end{array}\right) & =\left(\begin{array}{c}
-2 f(y)-x \\
2 g(-2 f(y)-x)-y
\end{array}\right)
\end{aligned}
$$

Clearly the map $T$ is completely determined by the choice of functions $f$ and $g$. This leads to the following definition.

Definition 3.1.1. Let $f, g: \mathbb{R} \rightarrow \mathbb{R}$ be $C^{1}$ functions with domain all of $\mathbb{R}$. $T:[f, g]$ is called the mapping associated to $f$ and $g$, and is given by

$$
T:[f, g]\left(\begin{array}{c}
x \\
y
\end{array}\right)=\left(\begin{array}{c}
-2 f(y)-x \\
2 g(-2 f(y)-x)-y
\end{array}\right) .
$$

Where $f$ and $g$ are understood from the context, we will write $T:[f, g]$ as simply $T$. 
The fixed point sets $\mathcal{F}$ and $\mathcal{G}$ of the maps $F$ and $G$ respectively will be very important in finding and classifying fixed points. We note that $\mathcal{F}$ is the graph of $y=-f(x)$ and $\mathcal{G}$ is the graph of $y=g(x)$. We have the following lemma relating fixed points for $T$ to intersections of $\mathcal{F}$ and $\mathcal{G}$.

Lemma 3.1.1. A point $(a, b)$ is a fixed point for $T$ if and only if $(a, b)$ is an intersection of $\mathcal{F}$ and $\mathcal{G}$.

Proof. If $(a, b)$ is an intersection of $\mathcal{F}$ and $\mathcal{G}$, then $F(a, b)=(a, b)$ and $G(a, b)=(a, b)$ so that $T(a, b)=G(F(a, b))=(a, b)$.

To prove the other direction, let $(a, b)$ be a fixed point for $T$ and assume that $(a, b)$ is not fixed by $F$. Then we have that $F(a, b)=\left(a+k_{1}, b\right)$, since $F$ is a horizontal reflection, and $T(a, b)=G(F(a, b))=\left(a+k_{1}, b+k_{2}\right)$, since $G$ s a vertical reflection. But $(a, b)$ is fixed by $T$, so we must have that $k_{1}=0$ and $k_{2}=0$, which implies that $F(a, b)=(a, b)$. But then $(a, b)=T(a, b)=G(F(a, b))=G(a, b)$, which proves that $(a, b)$ is also fixed by $G$. So since $(a, b)$ is fixed by both $F$ and $G$ then $(a, b)$ is an intersection of $\mathcal{F}$ and $\mathcal{G}$.

We will adopt the following intuitive conventions.

Definition 3.1.2. The region $\{y: y<g(x)\}$ will be called below $\mathcal{G}$, and the region $\{y: y>g(x)\}$ will be called above $\mathcal{G}$.

We note that the curve $\mathcal{F}$ is given parametrically by $(-f(t), t)$. Let $(a, b)$ be a point where $\mathcal{F}$ and $\mathcal{G}$ intersect.

Definition 3.1.3. We will say that $\mathcal{F}$ crosses $\mathcal{G}$ from below if there exists some $\epsilon>0$ such that the set $\{(-f(t), t): t \in(b-\epsilon, b)\}$ is below $\mathcal{G}$ and the set $\{(-f(t), t): t \in(b, b+\epsilon)\}$ is above $\mathcal{G}$. Similarly, we will say that $\mathcal{F}$ crosses $\mathcal{G}$ from above if there exists some $\epsilon>0$ such that the set $\{(-f(t), t): t \in(b-\epsilon, b)\}$ is above $\mathcal{G}$ and the set $\{(-f(t), t): t \in(b, b+\epsilon)\}$ is below $\mathcal{G}$. Further, if we say that $\mathcal{F}$ crosses $\mathcal{G}$, it will mean that either $\mathcal{F}$ crosses $\mathcal{G}$ from above or $\mathcal{F}$ crosses $\mathcal{G}$ from below.

Figure 3.2 should make these definitions clear. 


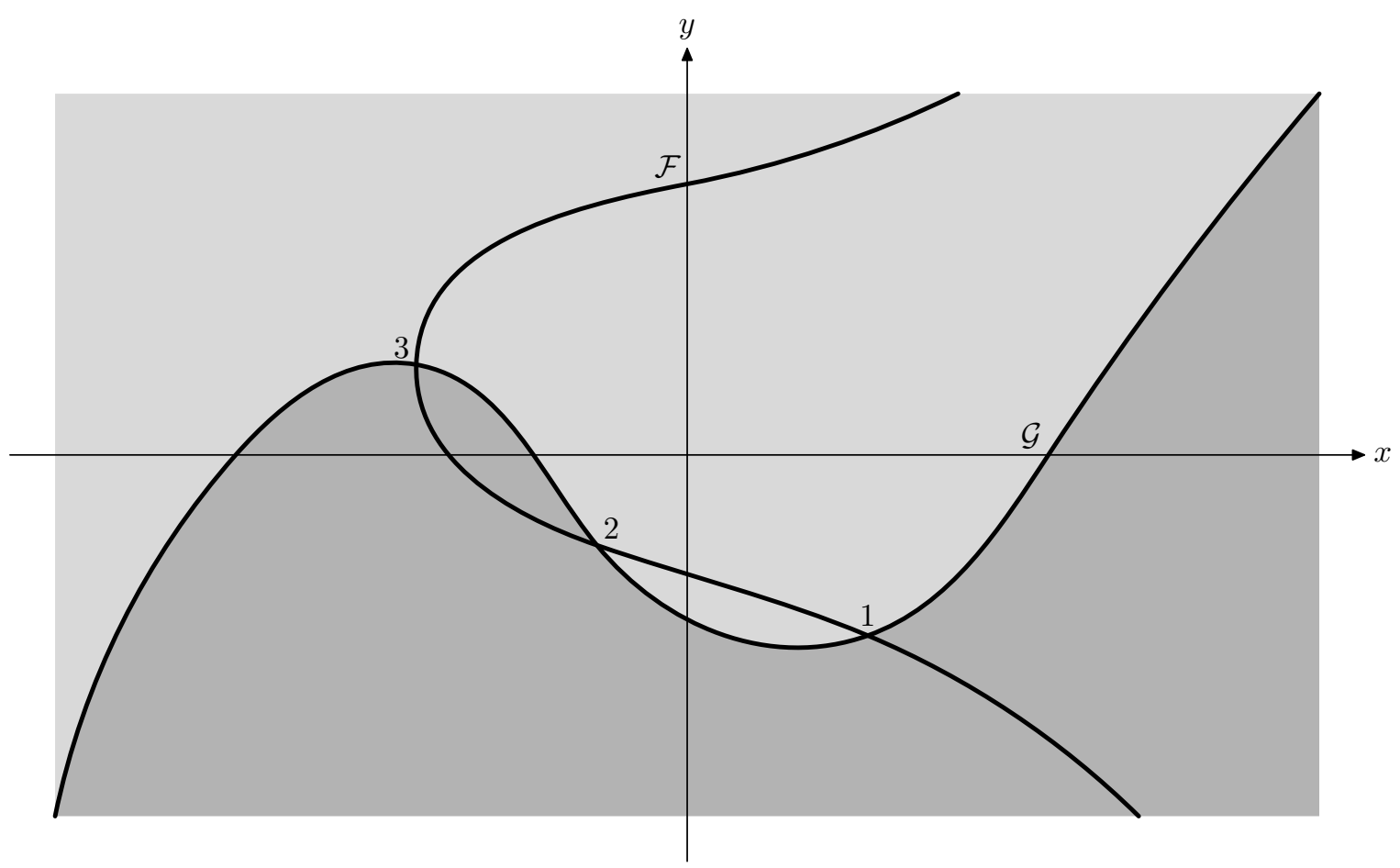

Figure 3.2: The lighter shaded region is above $\mathcal{G}$ and the darker shaded region shaded is below $\mathcal{G}$. At points 1 and $3, \mathcal{F}$ crosses $G$ from below, and at point $2, \mathcal{F}$ crosses $\mathcal{G}$ from above.

To begin the classification of fixed points, we make the following definitions.

Definition 3.1.4. A fixed point $p$ for a map $T:[f, g]$ is called a type A fixed point if $\mathcal{F}$ crosses $\mathcal{G}$ transversally from above at $p$.

Definition 3.1.5. A fixed point $p$ for a map $T:[f, g]$ is called a type B fixed point if $\mathcal{F}$ crosses $\mathcal{G}$ transversally from below at $p$.

For example, points 1 and 3 from Figure 3.2 are type B, and point 2 is type A. Combining these definitions with Lemma 3.1.1 we get the following lemma.

Lemma 3.1.2. Every fixed point of a map $T:[f, g]$ is either type $A$, type $B$, or a tangency of $\mathcal{F}$ and $\mathcal{G}$.

Now we state the Main Theorem of this thesis. 
Main Theorem. Let $f, g: \mathbb{R} \rightarrow \mathbb{R}$ be $C^{1}$ functions with domain all of $\mathbb{R}$, and consider the associated mapping $T:[f, g]$. Let $\mathcal{F}$ denote the graph of $x=-f(y)$ and $\mathcal{G}$ denote the graph of $y=g(x)$, and assume that all intersections of $\mathcal{F}$ and $\mathcal{G}$ are transverse. Then the following two statements are true.

1. If one of $f$ or $g$ is quadratic and the other is linear, then the map $T$ will admit a homoclinic point if and only if $\mathcal{F}$ and $\mathcal{G}$ intersect more than once.

2. If $f$ and $g$ are both quadratic, then the map $T$ will admit a homoclinic point if and only if $\mathcal{F}$ and $\mathcal{G}$ intersect more than once.

Next we look at relevant properties of mappings $T:[f, g]$.

\subsection{Properties of Mappings $T:[f, g]$}

$$
\begin{aligned}
& \text { For a given } f \text { and } g \text {, we may calculate } D T\left(\begin{array}{l}
x \\
y
\end{array}\right) \text { : } \\
& \qquad D T\left(\begin{array}{l}
x \\
y
\end{array}\right)=\left(\begin{array}{cc}
-1 & -2 f^{\prime}(y) \\
-2 g^{\prime}(-2 f(y)-x) & -4 g^{\prime}(-2 f(y)-x) f^{\prime}(y)-1
\end{array}\right)
\end{aligned}
$$

Let $(a, b)$ be a fixed point for $T$. By Lemma 3.1.1 this will be the case if and only if $(a, b)$ is a fixed point for both $F$ and $G$. This means that

$$
F\left(\begin{array}{l}
a \\
b
\end{array}\right)=\left(\begin{array}{c}
-2 f(b)-a \\
b
\end{array}\right)=\left(\begin{array}{l}
a \\
b
\end{array}\right) \Rightarrow-2 f(b)-a=a
$$

and that

$$
G\left(\begin{array}{l}
a \\
b
\end{array}\right)=\left(\begin{array}{c}
a \\
2 g(a)-b
\end{array}\right)=\left(\begin{array}{l}
a \\
b
\end{array}\right) \Rightarrow 2 g(a)-b=b .
$$

This leads to the following lemma. 
Lemma 3.2.1. For a fixed point $(a, b)$ we have

$$
D T\left(\begin{array}{l}
a \\
b
\end{array}\right)=\left(\begin{array}{cc}
-1 & -2 f^{\prime}(b) \\
-2 g^{\prime}(a) & -4 f^{\prime}(b) g^{\prime}(a)-1
\end{array}\right)
$$

Proof. Substitute $(a, b)$ into Equation (3.1) and use Equation (3.2).

To simplify notation, we will use the convention that if a fixed point $(a, b)$ is given, then $f^{\prime}$ will denote $f^{\prime}(b)$ and $g^{\prime}$ will denote $g^{\prime}(a)$. Using this notation, we have that

$$
D T\left(\begin{array}{l}
a \\
b
\end{array}\right)=\left(\begin{array}{cc}
-1 & -2 f^{\prime} \\
-2 g^{\prime} & -4 f^{\prime} g^{\prime}-1
\end{array}\right)
$$

This matrix has characteristic polynomial

$$
\lambda^{2}+\left(4 f^{\prime} g^{\prime}+2\right) \lambda+1
$$

which has eigenvalues

$$
\lambda_{ \pm}=-1-2 f^{\prime} g^{\prime} \pm 2 \sqrt{f^{\prime} g^{\prime}\left(f^{\prime} g^{\prime}+1\right)} .
$$

Since the map $T$ is area preserving, we have that $\lambda_{+} \lambda_{-}=1$. Therefore, a fixed point $(a, b)$ is hyperbolic if and only if the eigenvalues are real, which will occur if and only if $f^{\prime} g^{\prime}\left(f^{\prime} g^{\prime}+1\right)>0$. This proves the following lemma.

Lemma 3.2.2. A fixed point $(a, b)$ is hyperbolic if and only if $f^{\prime} g^{\prime}\left(f^{\prime} g^{\prime}+1\right)>0$, which will occur if either $f^{\prime} g^{\prime}<-1$ or $f^{\prime} g^{\prime}>0$.

We want to classify all possible different types of hyperbolic fixed points for $T$. To that end, we consider all the possible ways that $\mathcal{F}$ can cross $\mathcal{G}$.

So, let $(a, b)$ be a fixed point for a map $T=G \circ F$. We will denote the slopes of $\mathcal{F}$ and $\mathcal{G}$ at $(a, b)$ by $\mathcal{F}^{\prime}$ and $\mathcal{G}^{\prime}$, respectively. We note that $\mathcal{F}^{\prime}=\frac{-1}{f^{\prime}}$ and $\mathcal{G}^{\prime}=g^{\prime}$. Also, if either $f^{\prime}=0$ or $g^{\prime}=0$ then $f^{\prime} g^{\prime}=0$ and the fixed point will not be hyperbolic. So, in what follows we do not consider the cases where $f^{\prime}=0$ (ie. $\mathcal{F}$ has vertical slope) or $g^{\prime}=0$. 
Suppose that $\mathcal{F}$ crosses $\mathcal{G}$ from below, so that we are dealing with a type B fixed point, and $g^{\prime}>0$. Then there are two possibilities for $\mathcal{F}$.

Case 1) $\mathcal{F}$ crosses $\mathcal{G}$ from the region illustrated in Figure 3.3.

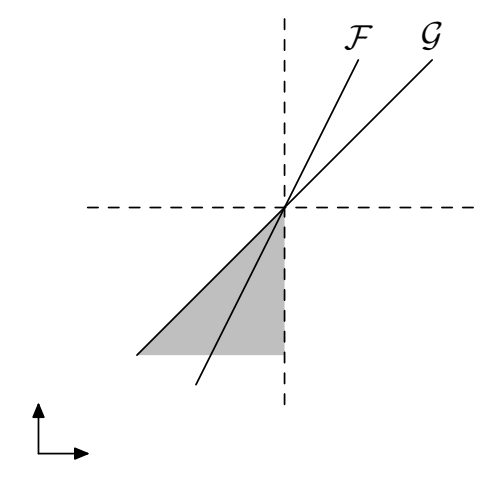

Figure 3.3: Case 1): not hyperbolic

In this case, $\mathcal{F}^{\prime} \in\left[\mathcal{G}^{\prime}, \infty\right) \Rightarrow g^{\prime} \leq \frac{-1}{f^{\prime}} \Rightarrow f^{\prime} g^{\prime} \geq-1$. Also, since $g^{\prime}>0$ and $f^{\prime}<0$, then $f^{\prime} g^{\prime}<0$. So, we have that $-1 \leq f^{\prime} g^{\prime}<0$, and so this fixed point is not hyperbolic.

Case 2) $\mathcal{F}$ crosses $\mathcal{G}$ from the region illustrated in Figure 3.4.

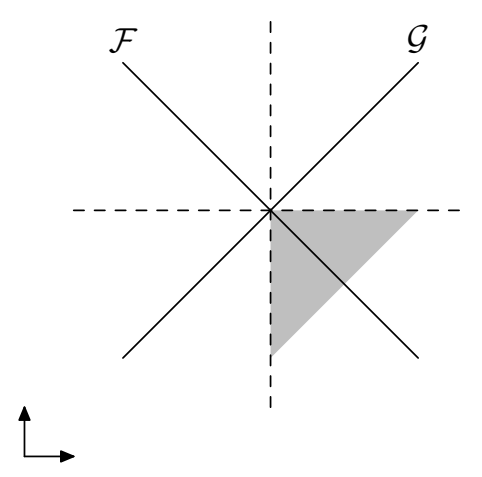

Figure 3.4: Case 2): the type B1 hyperbolic point

In this case, $\mathcal{F}^{\prime}<0 \Rightarrow f^{\prime}>0 \Rightarrow f^{\prime} g^{\prime}>0$. So, this fixed point is hyperbolic and we will call it type B1. 
Now, suppose that $\mathcal{F}$ crosses $\mathcal{G}$ from below, so that we are again dealing with a type B fixed point, and $g^{\prime}<0$. Again, there are two possibilities for $\mathcal{F}$.

Case 3) $\mathcal{F}$ crosses $\mathcal{G}$ from the region illustrated in Figure 3.5.

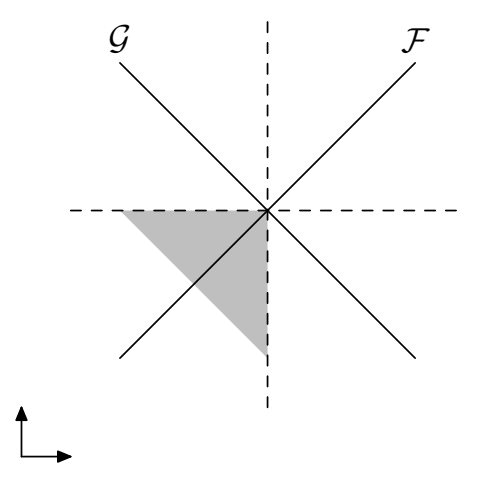

Figure 3.5: Case 3): the type B2 hyperbolic point

In this case, $\mathcal{F}^{\prime}>0 \Rightarrow f^{\prime}<0 \Rightarrow f^{\prime} g^{\prime}>0$, since $g^{\prime}<0$. So, this point is hyperbolic and we will call it type B2.

Case 4) $\mathcal{F}$ crosses $\mathcal{G}$ from the region illustrated in Figure 3.6.

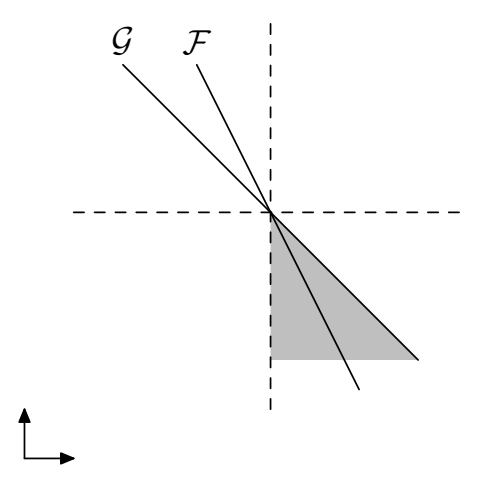

Figure 3.6: Case 4): not hyperbolic

In this case $\mathcal{F}^{\prime} \in\left(-\infty, \mathcal{G}^{\prime}\right] \Rightarrow \frac{-1}{f^{\prime}} \leq g^{\prime} \Rightarrow-1 \leq f^{\prime} g^{\prime}$. Also, since $f^{\prime}>0$ and $g^{\prime}<0$ then $f^{\prime} g^{\prime}<0$. So, we have that $-1 \leq f^{\prime} g^{\prime}<0$ and so this fixed point is not hyperbolic. 
Now, suppose that $\mathcal{F}$ crosses $\mathcal{G}$ from above, so that we are dealing with a type A fixed point, and $g^{\prime}>0$. There is only one possibility for $\mathcal{F}$.

Case 5) $\mathcal{F}$ crosses $\mathcal{G}$ from the region illustrated in Figure 3.7. In this case

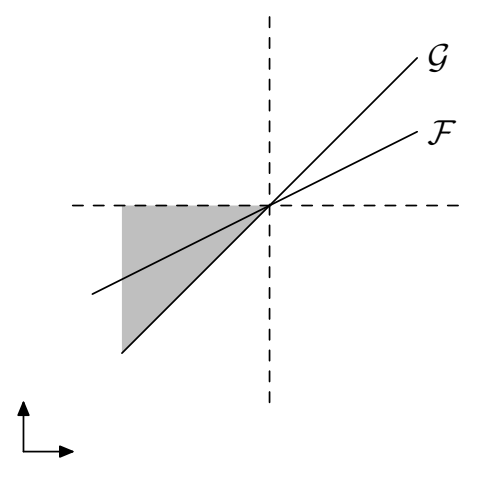

Figure 3.7: Case 5): the type A1 hyperbolic point

$\mathcal{F}^{\prime} \in\left(0, \mathcal{G}^{\prime}\right] \Rightarrow \frac{-1}{f^{\prime}} \leq g^{\prime} \Rightarrow-1 \geq f^{\prime} g^{\prime}$. So, if $\mathcal{F}^{\prime} \neq \mathcal{G}^{\prime}$ then this fixed point will be hyperbolic and we will call it type A1.

Now, suppose that $\mathcal{F}$ crosses $\mathcal{G}$ from above, so that we are again dealing with a type A fixed point, and $g^{\prime}<0$. Again, there is only one possibility for $\mathcal{F}$.

Case 6) $\mathcal{F}$ crosses $\mathcal{G}$ from the region illustrated in Figure 3.8. In this case

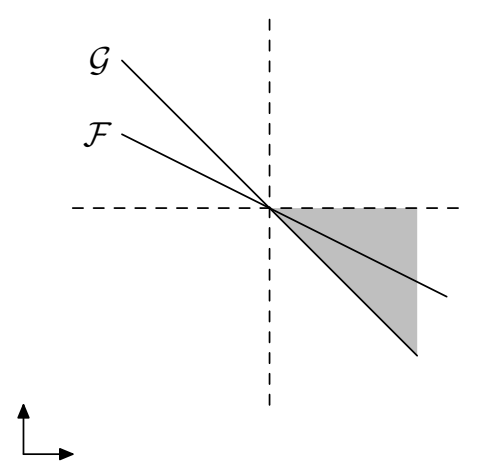

Figure 3.8: Case 6): the type A2 hyperbolic point 
$\mathcal{F}^{\prime} \in\left[\mathcal{G}^{\prime}, 0\right) \Rightarrow g^{\prime} \leq \frac{-1}{f^{\prime}} \Rightarrow f^{\prime} g^{\prime} \leq-1$. So, if $\mathcal{F}^{\prime} \neq \mathcal{G}^{\prime}$ then this fixed point will be hyperbolic and we will call it type A2.

Here is a summary of the hyperbolic fixed points.

Type B1 $\bullet g^{\prime}>0$

- $f^{\prime}>0$

- $f^{\prime} g^{\prime}>0$

- $\mathcal{F}$ crosses $\mathcal{G}$ from below

Type B2 $\bullet g^{\prime}<0$

- $f^{\prime}<0$

- $f^{\prime} g^{\prime}>0$

- $\mathcal{F}$ crosses $\mathcal{G}$ from below

Type A1 $\bullet g^{\prime}>0$

- $f^{\prime}<0$

- $f^{\prime} g^{\prime}<-1$

- $\mathcal{F}$ crosses $\mathcal{G}$ from above

Type A2 $\bullet g^{\prime}<0$

- $f^{\prime}>0$

- $f^{\prime} g^{\prime}<-1$

- $\mathcal{F}$ crosses $\mathcal{G}$ from above

The preceeding dicsussion leads to several lemmas.

Lemma 3.2.3. Type A fixed points are hyperbolic. 
Proof. Let $(a, b)$ be a type A fixed point. We have seen that if $g^{\prime}>0$ or $g^{\prime}<0$, then $(a, b)$ is hyperbolic. The only other case to consider is $g^{\prime}=0$, but this cannot happen at a type A fixed point, since if $g^{\prime}=0$ then $\mathcal{F}$ cannot cross $\mathcal{G}$ from above at $(a, b)$.

Lemma 3.2.4. Tangential fixed points are not hyperbolic.

Proof. Let $(a, b)$ be a fixed point at which $\mathcal{F}$ and $\mathcal{G}$ are tangent. Then at $(a, b)$ we have that $\frac{-1}{f^{\prime}}=g^{\prime}$ so that $f^{\prime} g^{\prime}=-1$. But then by Lemma 3.2.2, $(a, b)$ cannot be hyperbolic.

Lemma 3.2.5. Type A fixed points have positive eigenvalues.

Proof. If $(a, b)$ is a type A fixed point, then $f^{\prime} g^{\prime}<-1$. By Equation $(3.3), D T(a, b)$ has eigenvalues $\lambda_{ \pm}=-1-2 f^{\prime} g^{\prime} \pm 2 \sqrt{f^{\prime} g^{\prime}\left(f^{\prime} g^{\prime}+1\right)}$. Substituting $f^{\prime} g^{\prime}<-1$ into this equation yields two positive eigenvalues.

Lemma 3.2.6. Hyperbolic type B fixed points have negative eigenvalues.

Proof. If $(a, b)$ is a hyperbolic type B fixed point, then $f^{\prime} g^{\prime}>0$. By Equation $(3.3), D T(a, b)$ has eigenvalues $\lambda_{ \pm}=-1-2 f^{\prime} g^{\prime} \pm 2 \sqrt{f^{\prime} g^{\prime}\left(f^{\prime} g^{\prime}+1\right)}$. Substituting $f^{\prime} g^{\prime}>0$ into this equation yields two negative eigenvalues.

\subsection{A Closer Look at the Hyperbolic Fixed Points}

Let $(a, b)$ be a hyperbolic fixed point, and denote the stable and unstable manifolds of $(a, b)$ by $W^{s}(a, b)$ and $W^{u}(a, b)$ respectively. We will find the slopes of $\mathcal{F}, \mathcal{G}, W^{s}(a, b)$ and $W^{u}(a, b)$ at $(a, b)$. It is clear that $\mathcal{F}$ has slope $\frac{-1}{f^{\prime}}$ at $(a, b)$ and that $\mathcal{G}$ has slope $g^{\prime}$ at $(a, b)$.

To find the slopes of $W^{s}(a, b)$ and $W^{u}(a, b)$ at $(a, b)$ we recall that the eigenvalues of $D T(a, b)$ are given by

$$
\lambda_{ \pm}=-1-2 f^{\prime} g^{\prime} \pm 2 \sqrt{f^{\prime} g^{\prime}\left(f^{\prime} g^{\prime}+1\right)} .
$$


Without any more information about $f^{\prime}$ and $g^{\prime}$, we cannot determine which of $\lambda_{ \pm}$is the expanding eigenvalue and which is the contracting eigenvalue. We can, however, determine the slope of the eigenvectors corresponding to $\lambda_{+}$and $\lambda_{-}$.

$$
\begin{aligned}
& \operatorname{DT}(a, b)\left(\begin{array}{l}
\alpha \\
\beta
\end{array}\right)=\left(\begin{array}{c}
\lambda_{ \pm} \alpha \\
\lambda_{ \pm} \beta
\end{array}\right) \\
& \Rightarrow\left(\begin{array}{cc}
-1 & -2 f^{\prime} \\
-2 g^{\prime} & -4 f^{\prime} g^{\prime}-1
\end{array}\right)\left(\begin{array}{l}
\alpha \\
\beta
\end{array}\right)=\left(\begin{array}{c}
\lambda_{ \pm} \alpha \\
\lambda_{ \pm} \beta
\end{array}\right) \\
& \Rightarrow-\alpha-2 f^{\prime} \beta=\lambda_{ \pm} \alpha \\
& \Rightarrow \quad \text { the slope of the eigenvector is } \frac{\lambda_{ \pm}+1}{-2 f^{\prime}}
\end{aligned}
$$

We then calculate that

$$
\begin{aligned}
\frac{\lambda_{ \pm}+1}{-2 f^{\prime}} & =\frac{-2 f^{\prime} g^{\prime} \pm 2 \sqrt{f^{\prime} g^{\prime}\left(f^{\prime} g^{\prime}+1\right)}}{-2 f^{\prime}} \\
& =g^{\prime} \mp \frac{1}{f^{\prime}} \sqrt{f^{\prime} g^{\prime}\left(f^{\prime} g^{\prime}+1\right)}
\end{aligned}
$$

So, the slope of the eigenvector corresponding to $\lambda_{+}$is $g^{\prime}-\frac{1}{f^{\prime}} \sqrt{f^{\prime} g^{\prime}\left(f^{\prime} g^{\prime}+1\right)}$ and the slope of the eigenvector corresponding to $\lambda_{-}$is $g^{\prime}+\frac{1}{f^{\prime}} \sqrt{f^{\prime} g^{\prime}\left(f^{\prime} g^{\prime}+1\right)}$.

If $(a, b)$ is of type B1 or B2, then $f^{\prime} g^{\prime}>0$ so that $-1-2 f^{\prime} g^{\prime}<-1$. This means that $\lambda_{-}$is the expanding eigenvalue and $\lambda_{+}$is the contracting eigenvalue.

If $(a, b)$ is of type A1 or A2, then $f^{\prime} g^{\prime}<-1$ so that $-1-2 f^{\prime} g^{\prime}>1$. This means that $\lambda_{+}$is the expanding eigenvalue and $\lambda_{-}$is the contracting eigenvalue.

So, we have the following conclusion. If $(a, b)$ is of type $\mathrm{B} 1$ or $\mathrm{B} 2$, then

- $W^{u}$ has slope $g^{\prime}+\frac{1}{f^{\prime}} \sqrt{f^{\prime} g^{\prime}\left(f^{\prime} g^{\prime}+1\right)}$

- $W^{s}$ has slope $g^{\prime}-\frac{1}{f^{\prime}} \sqrt{f^{\prime} g^{\prime}\left(f^{\prime} g^{\prime}+1\right)}$

If $(a, b)$ is of type $\mathrm{A} 1$ or $\mathrm{A} 2$, then 
- $W^{u}$ has slope $g^{\prime}-\frac{1}{f^{\prime}} \sqrt{f^{\prime} g^{\prime}\left(f^{\prime} g^{\prime}+1\right)}$

- $W^{s}$ has slope $g^{\prime}+\frac{1}{f^{\prime}} \sqrt{f^{\prime} g^{\prime}\left(f^{\prime} g^{\prime}+1\right)}$

Now we will use this information to get a better picture of each hyperbolic fixed point.

\subsubsection{Type B1}

At a type B1 fixed point $(a, b)$, we have $f^{\prime}>0, g^{\prime}>0$, and $f^{\prime} g^{\prime}>0$. We also have the following slopes at the point $(a, b)$.

- $\mathcal{F}$ has slope $\frac{-1}{f^{\prime}}$

- $\mathcal{G}$ has slope $g^{\prime}$

- $W^{u}$ has slope $g^{\prime}+\frac{1}{f^{\prime}} \sqrt{f^{\prime} g^{\prime}\left(f^{\prime} g^{\prime}+1\right)}$

- $W^{s}$ has slope $g^{\prime}-\frac{1}{f^{\prime}} \sqrt{f^{\prime} g^{\prime}\left(f^{\prime} g^{\prime}+1\right)}$

Lemma 3.3.1. At a B1 fixed point the slope of $W^{u}$ is greater than the slope of $\mathcal{G}$.

Proof. This is clear since $f^{\prime}>0$ implies that $g^{\prime}+\frac{1}{f^{\prime}} \sqrt{f^{\prime} g^{\prime}\left(f^{\prime} g^{\prime}+1\right)}>g^{\prime}$.

Lemma 3.3.2. At a B1 fixed point, the slope of $W^{s}$ is negative and greater than the slope of $\mathcal{F}$.

Proof. To prove the first part, note that

$$
\begin{aligned}
g^{\prime}-\frac{1}{f^{\prime}} \sqrt{f^{\prime} g^{\prime}\left(f^{\prime} g^{\prime}+1\right)}<0 & \Longleftrightarrow f^{\prime} g^{\prime}<\sqrt{f^{\prime} g^{\prime}\left(f^{\prime} g^{\prime}+1\right)} \\
& \Longleftrightarrow\left(f^{\prime} g^{\prime}\right)^{2}<\left(f^{\prime} g^{\prime}\right)^{2}+f^{\prime} g^{\prime} \\
& \Longleftrightarrow 0<f^{\prime} g^{\prime}, \quad \text { which is true. }
\end{aligned}
$$


To prove the second part, note that

$$
\begin{aligned}
\frac{-1}{f^{\prime}}<g^{\prime}-\frac{1}{f^{\prime}} \sqrt{f^{\prime} g^{\prime}\left(f^{\prime} g^{\prime}+1\right)} & \Longleftrightarrow-1<f^{\prime} g^{\prime}-\sqrt{f^{\prime} g^{\prime}\left(f^{\prime} g^{\prime}+1\right)} \\
& \Longleftrightarrow \sqrt{f^{\prime} g^{\prime}\left(f^{\prime} g^{\prime}+1\right)}<f^{\prime} g^{\prime}+1 \\
& \Longleftrightarrow\left(f^{\prime} g^{\prime}\right)^{2}+f^{\prime} g^{\prime}<\left(f^{\prime} g^{\prime}\right)^{2}+2 f^{\prime} g^{\prime}+1 \\
& \Longleftrightarrow 0<f^{\prime} g^{\prime}+1, \quad \text { which is true. }
\end{aligned}
$$

The previous two lemmas allow us to get a picture of a B1 fixed point (Figure 3.9).

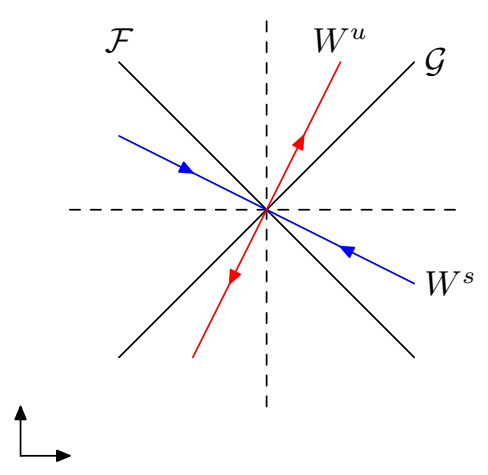

Figure 3.9: Type B1 fixed point

\subsubsection{Type B2}

At a type B2 fixed point $(a, b)$, we have $f^{\prime}<0, g^{\prime}<0$, and $f^{\prime} g^{\prime}>0$. We also have the following slopes at the point $(a, b)$.

- $\mathcal{F}$ has slope $\frac{-1}{f^{\prime}}$

- $\mathcal{G}$ has slope $g^{\prime}$

- $W^{u}$ has slope $g^{\prime}+\frac{1}{f^{\prime}} \sqrt{f^{\prime} g^{\prime}\left(f^{\prime} g^{\prime}+1\right)}$

- $W^{s}$ has slope $g^{\prime}-\frac{1}{f^{\prime}} \sqrt{f^{\prime} g^{\prime}\left(f^{\prime} g^{\prime}+1\right)}$ 
Lemma 3.3.3. At a B2 fixed point the slope of $W^{u}$ is less than the slope of $\mathcal{G}$.

Proof. This is clear since $f^{\prime}<0$ implies that $g^{\prime}+\frac{1}{f^{\prime}} \sqrt{f^{\prime} g^{\prime}\left(f^{\prime} g^{\prime}+1\right)}<g^{\prime}$.

Lemma 3.3.4. At a B2 fixed point, the slope of $W^{s}$ is positive and less than the slope of $\mathcal{F}$.

Proof. To prove the first part, note that

$$
\begin{aligned}
g^{\prime}-\frac{1}{f^{\prime}} \sqrt{f^{\prime} g^{\prime}\left(f^{\prime} g^{\prime}+1\right)}>0 & \Longleftrightarrow f^{\prime} g^{\prime}<\sqrt{f^{\prime} g^{\prime}\left(f^{\prime} g^{\prime}+1\right)} \\
& \Longleftrightarrow\left(f^{\prime} g^{\prime}\right)^{2}<\left(f^{\prime} g^{\prime}\right)^{2}+f^{\prime} g^{\prime} \\
& \Longleftrightarrow 0<f^{\prime} g^{\prime}, \quad \text { which is true. }
\end{aligned}
$$

To prove the second part, note that

$$
\begin{aligned}
g^{\prime}-\frac{1}{f^{\prime}} \sqrt{f^{\prime} g^{\prime}\left(f^{\prime} g^{\prime}+1\right)}<\frac{-1}{f^{\prime}} & \Longleftrightarrow-1<f^{\prime} g^{\prime}-\sqrt{f^{\prime} g^{\prime}\left(f^{\prime} g^{\prime}+1\right)} \\
& \Longleftrightarrow \sqrt{f^{\prime} g^{\prime}\left(f^{\prime} g^{\prime}+1\right)}<f^{\prime} g^{\prime}+1 \\
& \Longleftrightarrow\left(f^{\prime} g^{\prime}\right)^{2}+f^{\prime} g^{\prime}<\left(f^{\prime} g^{\prime}\right)^{2}+2 f^{\prime} g^{\prime}+1 \\
& \Longleftrightarrow 0<f^{\prime} g^{\prime}+1, \quad \text { which is true. }
\end{aligned}
$$

The previous two lemmas allow us to get a picture of a B2 fixed point (Figure 3.10).

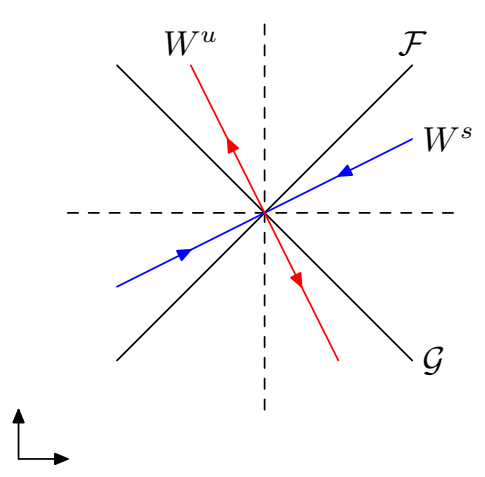

Figure 3.10: Type B2 fixed point 


\subsubsection{Type A1}

At a type $\mathrm{A} 1$ fixed point $(a, b)$, we have $f^{\prime}<0, g^{\prime}>0$, and $f^{\prime} g^{\prime}<-1$. We also have the following slopes at the point $(a, b)$.

- $\mathcal{F}$ has slope $\frac{-1}{f^{\prime}}$

- $\mathcal{G}$ has slope $g^{\prime}$

- $W^{u}$ has slope $g^{\prime}-\frac{1}{f^{\prime}} \sqrt{f^{\prime} g^{\prime}\left(f^{\prime} g^{\prime}+1\right)}$

- $W^{s}$ has slope $g^{\prime}+\frac{1}{f^{\prime}} \sqrt{f^{\prime} g^{\prime}\left(f^{\prime} g^{\prime}+1\right)}$

Lemma 3.3.5. At an $A 1$ fixed point the slope of $W^{u}$ is greater than the slope of $\mathcal{G}$.

Proof. This is clear since $f^{\prime}<0$ implies that $g^{\prime}-\frac{1}{f^{\prime}} \sqrt{f^{\prime} g^{\prime}\left(f^{\prime} g^{\prime}+1\right)}>g^{\prime}$.

Lemma 3.3.6. At an A1 fixed point, the slope of $W^{s}$ is positive and less than the slope of $\mathcal{F}$.

Proof. To prove the first part, note that

$$
\begin{aligned}
g^{\prime}+\frac{1}{f^{\prime}} \sqrt{f^{\prime} g^{\prime}\left(f^{\prime} g^{\prime}+1\right)}>0 & \Longleftrightarrow f^{\prime} g^{\prime}+\sqrt{f^{\prime} g^{\prime}\left(f^{\prime} g^{\prime}+1\right)}<0 \\
& \Longleftrightarrow f^{\prime} g^{\prime}<-\sqrt{f^{\prime} g^{\prime}\left(f^{\prime} g^{\prime}+1\right)} \\
& \Longleftrightarrow\left(f^{\prime} g^{\prime}\right)^{2}>\left(f^{\prime} g^{\prime}\right)^{2}+f^{\prime} g^{\prime} \\
& \Longleftrightarrow 0>f^{\prime} g^{\prime}, \quad \text { which is true. }
\end{aligned}
$$

To prove the second part, note that

$$
\begin{aligned}
g^{\prime}+\frac{1}{f^{\prime}} \sqrt{f^{\prime} g^{\prime}\left(f^{\prime} g^{\prime}+1\right)}<\frac{-1}{f^{\prime}} & \Longleftrightarrow-1<f^{\prime} g^{\prime}+\sqrt{f^{\prime} g^{\prime}\left(f^{\prime} g^{\prime}+1\right)} \\
& \Longleftrightarrow-\sqrt{f^{\prime} g^{\prime}\left(f^{\prime} g^{\prime}+1\right)}<f^{\prime} g^{\prime}+1 \\
& \Longleftrightarrow\left(f^{\prime} g^{\prime}\right)^{2}+f^{\prime} g^{\prime}>\left(f^{\prime} g^{\prime}\right)^{2}+2 f^{\prime} g^{\prime}+1 \\
& \Longleftrightarrow-1>f^{\prime} g^{\prime}, \quad \text { which is true. }
\end{aligned}
$$

The previous two lemmas allow us to get a picture of an A1 fixed point (Figure 3.11). 


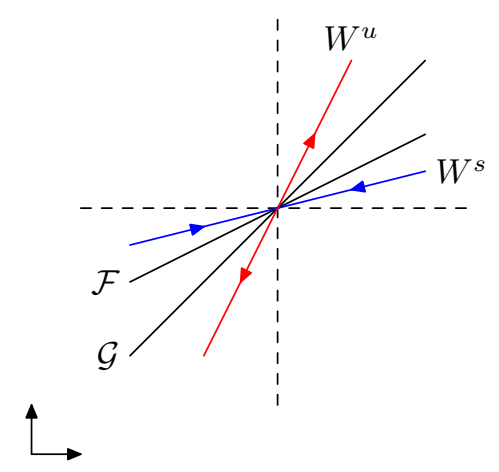

Figure 3.11: Type A1 fixed point

\subsubsection{Type A2}

At a type A2 fixed point $(a, b)$, we have $f^{\prime}>0, g^{\prime}<0$, and $f^{\prime} g^{\prime}<-1$. We also have the following slopes at the point $(a, b)$.

- $\mathcal{F}$ has slope $\frac{-1}{f^{\prime}}$

- $\mathcal{G}$ has slope $g^{\prime}$

- $W^{u}$ has slope $g^{\prime}-\frac{1}{f^{\prime}} \sqrt{f^{\prime} g^{\prime}\left(f^{\prime} g^{\prime}+1\right)}$

- $W^{s}$ has slope $g^{\prime}+\frac{1}{f^{\prime}} \sqrt{f^{\prime} g^{\prime}\left(f^{\prime} g^{\prime}+1\right)}$

Lemma 3.3.7. At an A2 fixed point the slope of $W^{u}$ is less than the slope of $\mathcal{G}$.

Proof. This is clear since $f^{\prime}>0$ implies that $g^{\prime}-\frac{1}{f^{\prime}} \sqrt{f^{\prime} g^{\prime}\left(f^{\prime} g^{\prime}+1\right)}<g^{\prime}$.

Lemma 3.3.8. At an A2 fixed point, the slope of $W^{s}$ is negative and greater than the slope of $\mathcal{F}$.

Proof. To prove the first part, note that

$$
\begin{aligned}
g^{\prime}+\frac{1}{f^{\prime}} \sqrt{f^{\prime} g^{\prime}\left(f^{\prime} g^{\prime}+1\right)}<0 & \Longleftrightarrow f^{\prime} g^{\prime}+\sqrt{f^{\prime} g^{\prime}\left(f^{\prime} g^{\prime}+1\right)}<0 \\
& \Longleftrightarrow f^{\prime} g^{\prime}<-\sqrt{f^{\prime} g^{\prime}\left(f^{\prime} g^{\prime}+1\right)} \\
& \Longleftrightarrow\left(f^{\prime} g^{\prime}\right)^{2}>\left(f^{\prime} g^{\prime}\right)^{2}+f^{\prime} g^{\prime} \\
& \Longleftrightarrow 0>f^{\prime} g^{\prime}, \quad \text { which is true. }
\end{aligned}
$$


To prove the second part, note that

$$
\begin{aligned}
g^{\prime}+\frac{1}{f^{\prime}} \sqrt{f^{\prime} g^{\prime}\left(f^{\prime} g^{\prime}+1\right)}>\frac{-1}{f^{\prime}} & \Longleftrightarrow-1<f^{\prime} g^{\prime}+\sqrt{f^{\prime} g^{\prime}\left(f^{\prime} g^{\prime}+1\right)} \\
& \Longleftrightarrow-\sqrt{f^{\prime} g^{\prime}\left(f^{\prime} g^{\prime}+1\right)}<f^{\prime} g^{\prime}+1 \\
& \Longleftrightarrow\left(f^{\prime} g^{\prime}\right)^{2}+f^{\prime} g^{\prime}>\left(f^{\prime} g^{\prime}\right)^{2}+2 f^{\prime} g^{\prime}+1 \\
& \Longleftrightarrow-1>f^{\prime} g^{\prime}, \quad \text { which is true. }
\end{aligned}
$$

The previous two lemmas allow us to get a picture of an A2 fixed point (Figure 3.12).

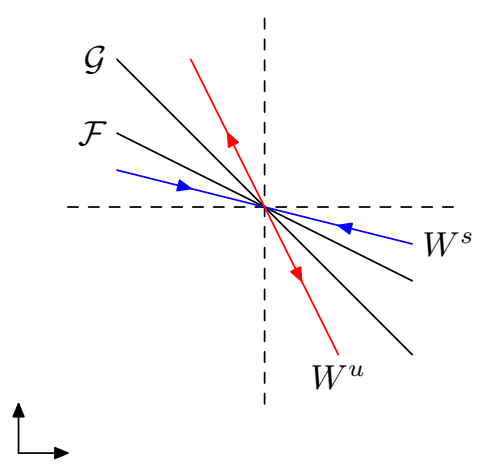

Figure 3.12: Type A2 fixed point

\subsection{On the Existence of Heteroclinic Connections}

In this section we look at some results involving heteroclinic connections. We let $T:[f, g]$ be defined as in Section 3.1 and let $p$ and $q$ be two hyperbolic fixed points for $T$. We will need the following definitions.

Definition 3.4.1. We say that $p$ and $q$ share a heteroclinic connection if one branch of the unstable manifold of one fixed point coincides with one branch of the stable manifold of the other fixed point. An example is shown in Figure 3.13. 


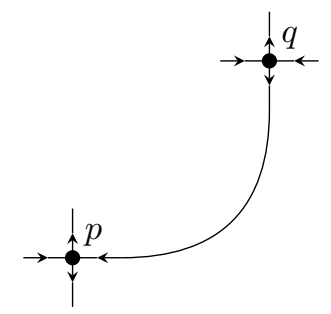

Figure 3.13: $p$ and $q$ share a heteroclinic connection.

Definition 3.4.2. We say that $p$ and $q$ admit a heteroclinic point if the unstable manifold of one fixed point intersects the stable manifold of the other fixed point. An example is shown in Figure 3.14 .

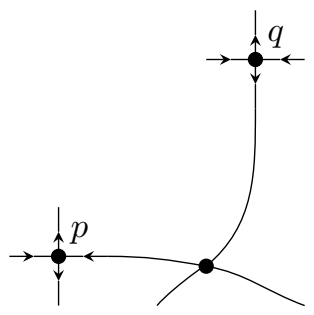

Figure 3.14: $p$ and $q$ admit a heteroclinic point.

We will prove the following theorem.

Theorem 3.4.1. A type A fixed point cannot share a heteroclinic connection with a type B fixed point.

Proof. Let A denote a type A fixed point, and let B denote a type B fixed point. By Lemma 3.2.3 we know that $\mathrm{A}$ is hyperbolic. We assume that $\mathrm{B}$ admits both a stable and unstable manifold, since otherwise there is no heteroclinic connection and we are done. Denote the stable and unstable manifolds of A by $W^{s}(\mathrm{~A})$ and $W^{u}(\mathrm{~A})$ respectively, and denote the stable and unstable manifolds of $\mathrm{B}$ by $W^{s}(\mathrm{~B})$ and $W^{u}(\mathrm{~B})$ respectively. We will show that $\mathrm{A}$ and $\mathrm{B}$ cannot share a heteroclinic connection. 
Since $T$ is $F$ and $G$ reversible, we may assume without loss of generality that one branch of $W^{u}(\mathrm{~B})\left(\right.$ call it $\left.W_{1}^{u}(\mathrm{~B})\right)$ coincides with one branch of $W^{s}(\mathrm{~A})$ (call it $\left.W_{1}^{s}(\mathrm{~A})\right)$. By lemma 3.2.6, B has negative eigenvalues, so the image under $T$ of $W_{1}^{u}(\mathrm{~B})$ is the other branch of $W^{u}(\mathrm{~B})$, which we will call $W_{2}^{u}(\mathrm{~B})$. By Lemma 3.2.5, A has positive eigenvalues, so the the image under $T$ of $W_{1}^{s}(\mathrm{~A})$ is again $W_{1}^{s}(\mathrm{~A})$.

So if there is a heteroclinic connection between $\mathrm{B}$ and $\mathrm{A}$, then we have that $W_{1}^{u}(\mathrm{~B})$ coincides with $W_{1}^{s}(\mathrm{~A})$. But the image under $T$ of this heteroclinic connection is another heteroclinic connection in which $W_{2}^{u}(\mathrm{~B})$ coincides with $W_{1}^{s}(\mathrm{~A})$. See Figure 3.15. This is clearly a contradiction since the stable manifold of a hyperbolic fixed point is a unique curve.

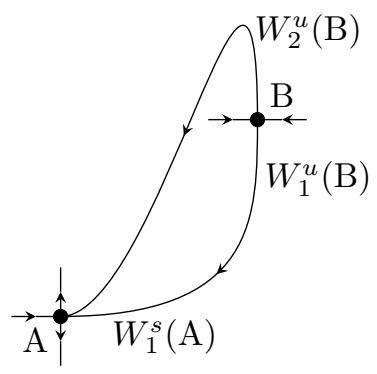

Figure 3.15: A heteroclinic connection between type A and type B fixed points contradicts the uniqueness of $W^{s}(\mathrm{~A})$.

\subsection{On The Existence of Homoclinic Points}

In this section we prove the following theorem.

Theorem 3.5.1. Let $f, g: \mathbb{R} \rightarrow \mathbb{R}$ be $C^{1}$ functions with domain all of $\mathbb{R}$, and consider the associated mapping $T:[f, g]$. If the following conditions are satisfied, then $T$ admits a homoclinic 
point.

1. There is at least one type A fixed point that is not part of a heteroclinic connection.

2. At least one of $f$ or $g$ has either a global maximum or a global minimum.

This theorem has the following corollary.

Corollary 3.5.1. Let $f, g: \mathbb{R} \rightarrow \mathbb{R}$ be $C^{1}$ functions with domain all of $\mathbb{R}$, and consider the associated mapping $T:[f, g]$. Let $\mathcal{F}$ denote the graph of $x=-f(y)$ and $\mathcal{G}$ denote the graph of $y=g(x)$, and assume that all intersections of $\mathcal{F}$ and $\mathcal{G}$ are transverse. If the following conditions are satisfied, then $T$ admits a homoclinic point.

1. There is exactly one type A fixed point.

2. At least one of $f$ or $g$ has either a global maximum or a global minimum.

If we assume for the moment that Theorem 3.5.1 is true, then we can prove the corollary as follows.

Proof. Denote the type A fixed point by $p$. By hypothesis, any other fixed points are type B, and $p$ cannot share a heteroclinic connection with these by Theorem 3.4.1. So, the hypothesis of Theorem 3.5.1 is satisfied. This proves the corollary.

In order to prove Theorem 3.5.1, we will need to consider eight separate cases. If we let $p$ denote a type A fixed point which is not part of any heteroclinic connection, then the eight cases are as follows.

1. $p$ is type $\mathrm{A} 2$ and $g$ has a global maximum.

2. $p$ is type A1 and $g$ has a global maximum.

3. $p$ is type $\mathrm{A} 1$ and $g$ has a global minimum.

4. $p$ is type A2 and $g$ has a global minimum. 
5. $p$ is type $\mathrm{A} 1$ and $f$ has a global minimum.

6. $p$ is type A2 and $f$ has a global maximum.

7. $p$ is type $\mathrm{A} 2$ and $f$ has a global minimum.

8. $p$ is type A1 and $f$ has a global maximum.

Cases 1, 2, 3, and 4 are illustrated in Figure 3.16, and cases 5, 6, 7, and 8 are illustrated in Figure 3.17 .

To facilitate proving Theorem 3.5.1, we will show that all eight cases are conjugate to each other.

The geometry of the conjugacies is fairly clear from Figures 3.16 and 3.17. Case 1 will be conjugate to case 2 via a reflection in the vertical axis. Case 2 will be conjugate to case 4 via a reflection in the horizontal axis, and case 4 will be conjugate to case 3 via a reflection in the vertical axis. So we have, with a slight abuse of notation and $\sim$ denoting conjugacy, that

Case $1 \sim$ Case $2 \sim$ Case $4 \sim$ Case 3 .

Similarly, Case 5 will be conjugate to case 6 via a reflection in the vertical axis. Case 6 will be conjugate to case 8 via a reflection in the horizontal axis, and case 8 will be conjugate to case 7 via a reflection in the vertical axis. So we have that

Case $5 \sim$ Case $6 \sim$ Case $8 \sim$ Case 7 .

To complete the chain, we need to show that that case 1 is conjugate to case 5 .

We will prove the following lemma.

Lemma 3.5.1. Let $i$ and $j$ be chosen from $\{1,2,3,4,5,6,7,8\}$. If a map $T:[f, g]$ admits a case $i$ situation, then there exist $f_{1}$ and $g_{1}$ such that $T_{1}:\left[f_{1}, g_{1}\right]$ is topologically conjugate to $T:[f, g]$ and $T_{1}:\left[f_{1}, g_{1}\right]$ admits a case $j$ situation .

The following lemma shows that cases 1 and 2 are topologically conjugate. 


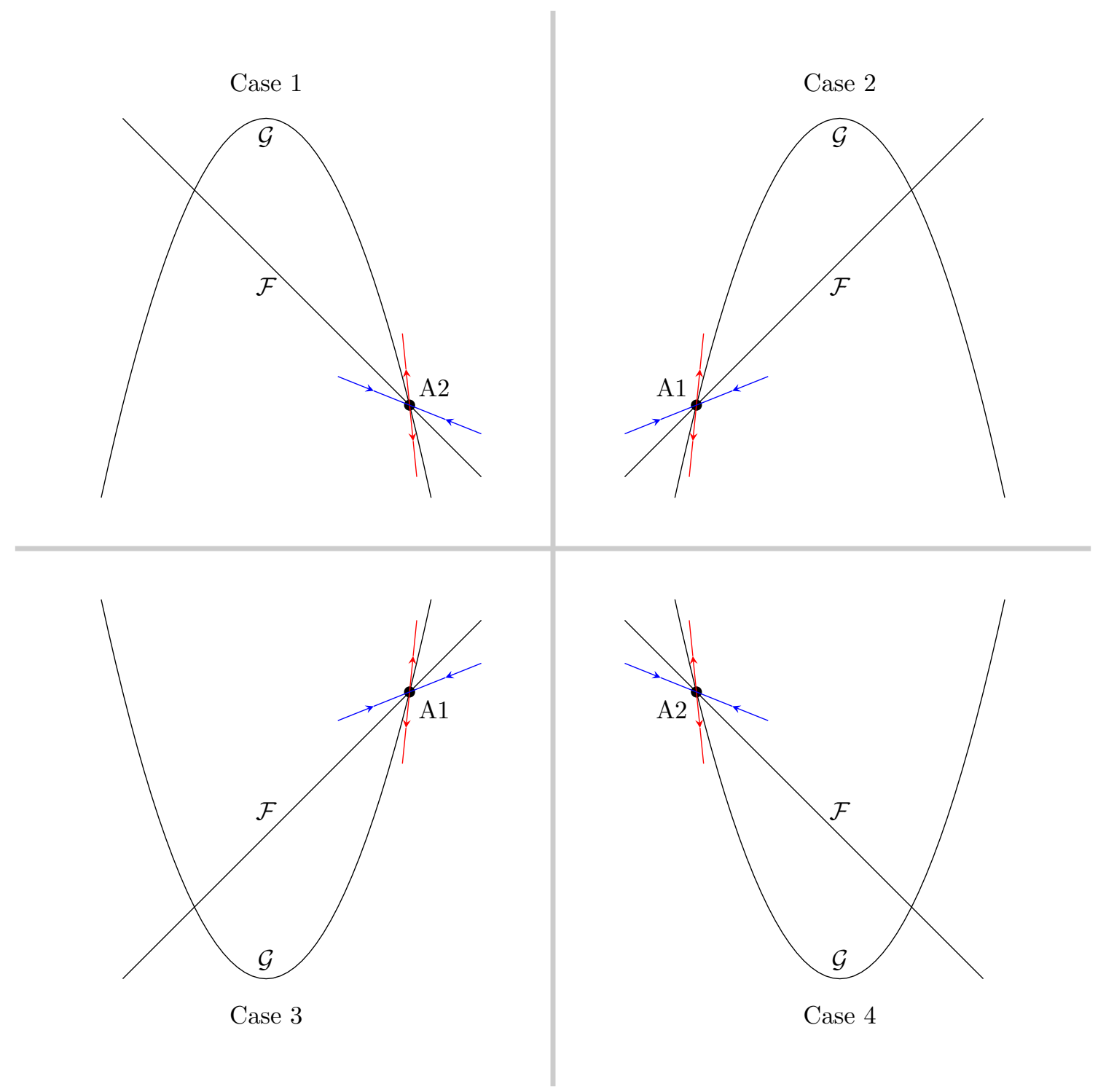

Figure 3.16: Cases 1, 2, 3, and 4. The red arrows indicate the initial slope of the unstable manifold of the fixed point, and the blue arrows indicate the initial slope of the stable manifold of the fixed point. 


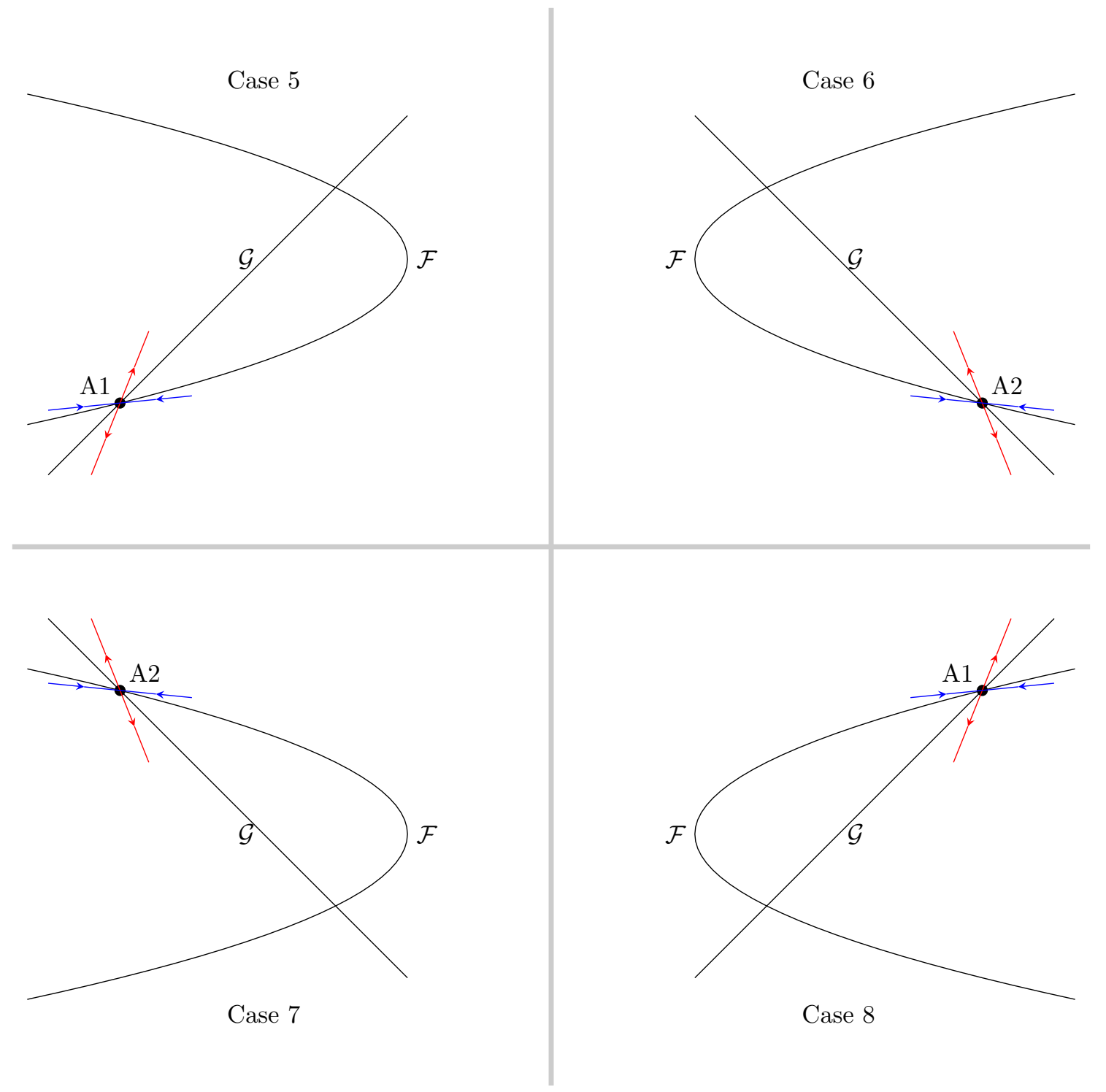

Figure 3.17: Cases 5, 6, 7, and 8. The red arrows indicate the initial slope of the unstable manifold of the fixed point, and the blue arrows indicate the initial slope of the stable manifold of the fixed point. 
Lemma 3.5.2. Suppose a map $T:[f, g]$ admits a case 1 situation, so that $T$ has a type A2 fixed point $(a, b)$ and $g$ has a global maximum. Then $T:[f, g]$ is topologically conjugate via a reflection in the vertical axis to $T_{1}:\left[f_{1}, g_{1}\right]$ where $f_{1}(y)=-f(y)$ and $g_{1}(x)=g(-x)$. Further, $T_{1}:\left[f_{1}, g_{1}\right]$ admits a case 2 situation, so that $T_{1}$ has a type A1 fixed point at $\left(a_{1}, b_{1}\right)=(-a, b)$ and $g_{1}$ has a global maximum.

Proof. Let $R$ denote a reflection in the vertical axis, so that $R(x, y)=(-x, y)$. Then

$$
\begin{aligned}
R \circ T \circ R^{-1}\left(\begin{array}{l}
x \\
y
\end{array}\right) & =R \circ T\left(\begin{array}{c}
-x \\
y
\end{array}\right) \\
& =R\left(\begin{array}{c}
-2 f(y)+x \\
2 g(-2 f(y)+x)-y
\end{array}\right) \\
& =\left(\begin{array}{c}
2 f(y)-x \\
2 g(-2 f(y)+x)-y
\end{array}\right) \\
& =\left(\begin{array}{c}
-2 f_{1}(y)-x \\
2 g_{1}\left(-2 f_{1}(y)-x\right)-y
\end{array}\right) \\
& =T_{1}\left(\begin{array}{l}
x \\
y
\end{array}\right)
\end{aligned}
$$

So $T \sim T_{1}$ via $R$. We calculate that $T_{1}\left(a_{1}, b_{1}\right)=\left(a_{1}, b_{1}\right)$ and it is clear from Figure 3.16 that $\left(a_{1}, b_{1}\right)$ is a type $\mathrm{A} 1$ fixed point. It is also clear that $g_{1}$ has a global maximum, since $g_{1}(x)=g(-x)$.

The following lemma shows that cases 5 and 6 are topologically conjugate.

Lemma 3.5.3. Suppose a map $T:[f, g]$ admits a case 5 situation, so that $T$ has a type A1 fixed point $(a, b)$ and $f$ has a global minimum. Then $T:[f, g]$ is topologically conjugate via a reflection in the vertical axis to $T_{1}:\left[f_{1}, g_{1}\right]$ where $f_{1}(y)=-f(y)$ and $g_{1}(x)=g(-x)$. Further, $T_{1}:\left[f_{1}, g_{1}\right]$ admits a case 6 situation, so that $T_{1}$ has a type A2 fixed point at $\left(a_{1}, b_{1}\right)=(-a, b)$ and $f_{1}$ has a 
global maximum.

Proof. Let $R$ denote a reflection in the vertical axis, so that $R(x, y)=(-x, y)$. Then

$$
\begin{aligned}
R \circ T \circ R^{-1}\left(\begin{array}{l}
x \\
y
\end{array}\right) & =R \circ T\left(\begin{array}{c}
-x \\
y
\end{array}\right) \\
& =R\left(\begin{array}{c}
-2 f(y)+x \\
2 g(-2 f(y)+x)-y
\end{array}\right) \\
& =\left(\begin{array}{c}
2 f(y)-x \\
2 g(-2 f(y)+x)-y
\end{array}\right) \\
& =\left(\begin{array}{c}
-2 f_{1}(y)-x \\
2 g_{1}\left(-2 f_{1}(y)-x\right)-y
\end{array}\right) \\
& =T_{1}\left(\begin{array}{l}
x \\
y
\end{array}\right)
\end{aligned}
$$

So $T \sim T_{1}$ via $R$. We calculate that $T_{1}\left(a_{1}, b_{1}\right)=\left(a_{1}, b_{1}\right)$ and it is clear from Figure 3.17 that $\left(a_{1}, b_{1}\right)$ is a type A2 fixed point. It is also clear that $f_{1}$ has a global maximum, since $f_{1}(y)=-f(y)$

The proofs that Case $2 \sim$ Case 4 , Case $4 \sim$ Case 3, Case $6 \sim$ Case 8 , and Case $8 \sim$ Case 7 are all very similar to the proofs of Lemma 3.5.2 and Lemma 3.5.3 and will be omitted. The proof that Case $1 \sim$ Case 5, however, is somewhat different, and so we state and prove the following lemma.

Lemma 3.5.4. Suppose a map $T:[f, g]$ admits a case 1 situation, so that $T$ has a type A2 fixed point $(a, b)$ and $g$ has a global maximum. Then $T:[f, g]$ is topologically conjugate to $T_{1}:\left[f_{1}, g_{1}\right]$ where $f_{1}(y)=-g(-y)$ and $g_{1}(x)=f(x)$. Further, $T_{1}:\left[f_{1}, g_{1}\right]$ admits a case 5 situation, so that $T_{1}$ has a type $A 1$ fixed point at $\left(a_{1}, b_{1}\right)=(b,-a)$ and $f_{1}$ has a global minimum.

Proof. First, we will show that $T:[f, g] \sim\left(T_{1}:\left[f_{1}, g_{1}\right]\right)^{-1}$ via a 90 degree clockwise rotation, ie. that $T$ is conjugate to the inverse of the map $T_{1}:\left[f_{1}, g_{1}\right]$. Second, we note that $T_{1} \sim T_{1}^{-1}$, 
which will give us the chain of conjugacies $T \sim T_{1}^{-1} \sim T_{1}$. Third, we verify that $\left(a_{1}, b_{1}\right)$ is a type A1 fixed point and that $f_{1}$ has a global minimum.

Let $R$ denote a 90 degree clockwise rotation, so that $R(x, y)=(y,-x)$. Then $R^{-1}(x, y)=(-y, x)$. Then

$$
\begin{aligned}
R \circ T \circ R^{-1}\left(\begin{array}{l}
x \\
y
\end{array}\right) & =R \circ T\left(\begin{array}{c}
-y \\
x
\end{array}\right) \\
& =R\left(\begin{array}{c}
-2 f(x)+y \\
2 g(-2 f(x)+y)-x
\end{array}\right) \\
& =\left(\begin{array}{c}
2 g(-2 f(x)+y)-x \\
2 f(x)-y
\end{array}\right) \\
& =\left(\begin{array}{c}
-2 f_{1}\left(2 g_{1}(x)-y\right)-x \\
2 g_{1}(x)-y
\end{array}\right) \\
& =T_{1}^{-1}\left(\begin{array}{l}
x \\
y
\end{array}\right)
\end{aligned}
$$

We note that $T_{1}^{-1}$ is a vertical reflection in the curve $y=g_{1}(x)$ followed by a horizontal reflection in the curve $x=f_{1}(y)$.

To show that $T_{1} \sim T_{1}^{-1}$, we note that $T_{1}^{-1}$ is the composition of two involutions, and that any such map must be conjugate to its own inverse. To see this, let $G=S_{1} \circ S_{2}$, where $S_{1}$ and $S_{2}$ are involutions. It is clear that $G^{-1}=S_{2} \circ S_{1}$, and that

$$
\begin{aligned}
S_{1} \circ G \circ S_{1} & =S_{1} \circ\left(S_{1} \circ S_{2}\right) \circ S_{1} \\
& =\left(S_{1} \circ S_{1}\right) \circ S_{2} \circ S_{1} \\
& =S_{2} \circ S_{1} \\
& =G^{-1}
\end{aligned}
$$


We have that $T_{1}$ is a horizontal reflection in the curve $x=f_{1}(y)$ followed by a vertical reflection in the curve $y=g_{1}(x)$. We may calculate that $T_{1}\left(a_{1}, b_{1}\right)=\left(a_{1}, b_{1}\right)$ and it is clear from Figure 3.17 that $\left(a_{1}, b_{1}\right)$ is a type A1 fixed point. Further, $f_{1}$ must have a global minimum since $g(y)$ has a global maximum and $f_{1}(y)=-g(-y)$.

Now we may prove Lemma 3.5.1.

Proof of Lemma 3.5.1. We have shown that

Case $7 \sim$ Case $8 \sim$ Case $6 \sim$ Case $5 \sim$ Case $1 \sim$ Case $2 \sim$ Case $4 \sim$ Case 3.

Now we prove Theorem 3.5.1.

Proof of Theorem 3.5.1. Denote the type A fixed point that is not part of a heteroclinic connection by $p$. We must prove the theorem for the previously mentioned eight cases.

By Lemma 3.5.1 we know that all eight cases are conjugate to each other. We will prove the theorem for case 5 , and this will prove it for all cases. The case 5 situation is illustrated in the top left picture of Figure 3.17.

We denote the type A1 fixed point by $p$. The key idea of the proof is to show that $W^{u}(p)$ must cross either $\mathcal{F}$ or $\mathcal{G}$. Since $T$ is both $F$ and $G$ reversible, then the results of Section 2.3 guarantee that such a crossing must be a homoclinic point.

Since $f$ has a global minimum, then $\mathcal{F}$ has a global 'rightward maximum', ie. there exists $M$ such that $-f(y) \leq M$ for all $y$. By the slope arguments of Section 3.3, there is a branch of $W^{u}(p)$ that starts to the left of $\mathcal{F}$ and above $\mathcal{G}$. Call this branch $W_{1}^{u}(p)$ and let $w \in W_{1}^{u}(p)$. By hypothesis, $p$ is not part of any heteroclinic connection, so any heteroclinic point on $W_{1}^{u}(p)$ must be isolated. Therefore, there exist points on $W_{1}^{u}(p)$ that are not heteroclinic points, and we may choose $w$ to be such a point. $T^{n}(w) \in W_{1}^{u}(p)$ for all $n \in \mathbb{Z}$ since the A1 fixed point has positive eigenvalues. We also have that as $n$ increases, $T^{n}(w)$ moves farther along $W_{1}^{u}(p)$. 
Let $T^{n}(w)=\left(\begin{array}{l}x_{n} \\ y_{n}\end{array}\right)$ and consider the following three statements.

S1: $\left\{x_{n}\right\}$ is a nondecreasing sequence.

S2: $\left\{x_{n}\right\}$ has a limit.

S3: $\left\{y_{n}\right\}$ is a nondecreasing sequence.

We will prove the theorem by showing the following

- If at least one of $\mathrm{S} 1, \mathrm{~S} 2$, and $\mathrm{S} 3$ is false, then $T$ will admit a homoclinic point.

- If all of S1, S2, and S3 are true, then we get a contradiction.

Suppose that $\mathrm{S} 1$ is false. In this case, we have that $x_{k}<x_{k-1}$ for some $k$, so that $T^{k}(w)$ is to the left of $T^{k-1}(w)$. Then $T^{k-1}(w)$ must have been to the right of $\mathcal{F}$, so that $W_{1}^{u}(p)$ must have crossed $\mathcal{F}$. This crossing will be a homoclinic point.

Suppose that $\mathrm{S} 2$ is false. If $\mathrm{S} 1$ is also false, then $T$ will have a homoclinic point, so we assume that $\mathrm{S} 1$ is true. If the sequence $\left\{x_{n}\right\}$ does not have a limit, then it must increase without bound. Thus, $T^{n}(w)$ will eventually be to the right of $\mathcal{F}$, which implies that $W_{1}^{u}(p)$ must have crossed $\mathcal{F}$. This crossing will be a homoclinic point.

Suppose that S3 is false. In this case we have that $y_{n}<y_{n-1}$, so that $T^{n}(w)$ is below $T^{n-1}(w)$. Then $T^{n}(w)$ must be below $\mathcal{G}$, so that $W_{1}^{u}(p)$ must have crossed $\mathcal{G}$. This crossing will be a homoclinic point.

Suppose that S1, S2, and S3 are all true. We calculate that

$$
T\left(\begin{array}{c}
x_{n} \\
y_{n}
\end{array}\right)=\left(\begin{array}{c}
-2 f\left(y_{n}\right)-x_{n} \\
2 g\left(-2 f\left(y_{n}\right)-x_{n}\right)-y_{n}
\end{array}\right)=\left(\begin{array}{c}
x_{n+1} \\
y_{n+1}
\end{array}\right)
$$

so we have that

$$
y_{n+1}=2 g\left(x_{n+1}\right)-y_{n} \Longrightarrow y_{n+1}+y_{n}=2 g\left(x_{n+1}\right) .
$$

Then $y_{n+1}+y_{n}$ must approach a limit because $\left\{x_{n}\right\}$ has a limit and $g$ is defined for all $\mathbb{R}$. So, the sequence $\left\{y_{n}\right\}$ is nondecreasing and has the property that the sum of two consecturive entries 
approaches a limit. Therefore $\left\{y_{n}\right\}$ must converge, since if it did not, then it could not have the property that $y_{n+1}+y_{n}$ approaches a limit. Since both $\left\{x_{n}\right\}$ and $\left\{y_{n}\right\}$ converge, then $T^{n}(w)$ approaches a limit. This limit must be a fixed point for $T$, which means that $w$ must be on the stable manifold of this fixed point. However, this contradicts the choice of $w$.

This proves the theorem.

\subsection{Proof of the Main Theorem}

Main Theorem. Let $f, g: \mathbb{R} \rightarrow \mathbb{R}$ be $C^{1}$ functions with domain all of $\mathbb{R}$, and consider the associated mapping $T:[f, g]$. Let $\mathcal{F}$ denote the graph of $x=-f(y)$ and $\mathcal{G}$ denote the graph of $y=g(x)$, and assume that all intersections of $\mathcal{F}$ and $\mathcal{G}$ are transverse. Then the following two statements are true.

1. If one of $f$ or $g$ is quadratic and the other is linear, then the map $T$ will admit a homoclinic point if and only if $\mathcal{F}$ and $\mathcal{G}$ intersect more than once.

2. If $f$ and $g$ are both quadratic, then the map $T$ will admit a homoclinic point if and only if $\mathcal{F}$ and $\mathcal{G}$ intersect more than once.

Proof. We need to consider eight cases.

1. One of $f$ or $g$ is quadratic and the other is linear and $\mathcal{F}$ and $\mathcal{G}$ do not intersect.

2. Both $f$ and $g$ are quadratic and $\mathcal{F}$ and $\mathcal{G}$ do not intersect.

3. One of $f$ or $g$ is quadratic and the other is linear and $\mathcal{F}$ and $\mathcal{G}$ intersect exactly once.

4. Both $f$ and $g$ are quadratic and $\mathcal{F}$ and $\mathcal{G}$ intersect exactly once.

5. One of $f$ or $g$ is quadratic and the other is linear and $\mathcal{F}$ and $\mathcal{G}$ intersect exactly twice.

6. Both $f$ and $g$ are quadratic and $\mathcal{F}$ and $\mathcal{G}$ intersect exactly twice. 
7. Both $f$ and $g$ are quadratic and $\mathcal{F}$ and $\mathcal{G}$ intersect exactly three times.

8. Both $f$ and $g$ are quadratic and $\mathcal{F}$ and $\mathcal{G}$ intersect exactly four times.

For cases $1,2,3$, and 4 we want to show that $T$ will not have a homoclinic point, and for cases $5,6,7$, and 8 we want to show that $T$ will have a homoclinic point. We will see that not all cases satisfy the hypothesis of the theorem.

For cases 1 and 2, it is clear by Lemma 3.1.1 that the map $T$ will have no fixed points, and so there can be no homoclinic points.

For case 3 , we need to consider the different ways in which $\mathcal{F}$ and $\mathcal{G}$ can intersect exactly once. These are illustrated in Figure 3.18.

Denote the intersection point by $(a, b)$. The situations where $\mathcal{F}$ and $\mathcal{G}$ are tangent at $(a, b)$, as in the top two diagrams in Figure 3.18, are ruled out by hypothesis.

If $\mathcal{F}$ and $\mathcal{G}$ intersect as in the bottom two diagrams in Figure 3.18, then either $f^{\prime}(b)=0$ or $g^{\prime}(a)=0$. In either case we have that $f^{\prime}(b) g^{\prime}(a)=0$, and so by Lemma 3.2.2 $(a, b)$ is not a hyperbolic fixed point.

Let us take a closer look at what happens in this case. Using translation and the methods of Lemma 3.5.1, we find that any situation as in the bottom two diagrams in Figure 3.18 will be conjugate to $T:[f, g]$ with $f(x)=0$ and $g(x)=a x^{2}+b x$, where $a \neq 0 . T:[f, g]$ will have a fixed point at $(0,0)$ with a double -1 eigenvalue. Further, we have that

$$
T\left(\begin{array}{l}
x \\
y
\end{array}\right)=\left(\begin{array}{c}
-x \\
2 a x^{2}-2 b x-y
\end{array}\right) \quad \text { and } \quad T^{2}\left(\begin{array}{l}
x \\
y
\end{array}\right)=\left(\begin{array}{c}
x \\
4 b x+y
\end{array}\right)
$$

so that every vertical line is invariant under $T^{2}$. Clearly there can be no homoclinic point in this situation.

So for case 3 , the map $T$ will not have a homoclinic point.

For case 4 , we note that the only way $\mathcal{F}$ and $\mathcal{G}$ can intersect exactly once is for the intersection point to be a tangency of $\mathcal{F}$ and $\mathcal{G}$, as in Figure 3.19. So, case 4 is ruled out by hypothesis. 


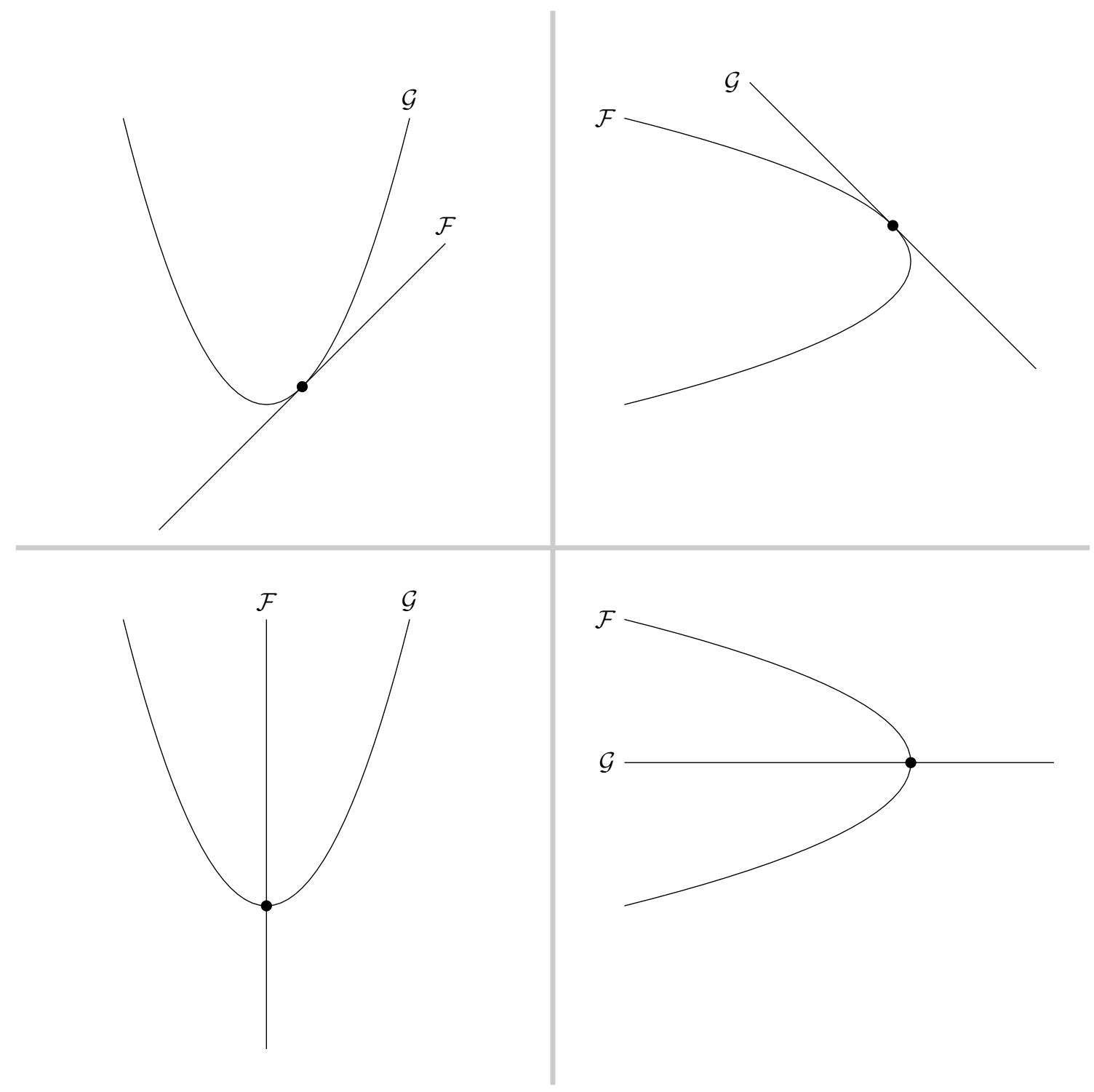

Figure 3.18: Four ways that $\mathcal{F}$ and $\mathcal{G}$ can intersect exactly once when one is linear and the other is quadratic. 


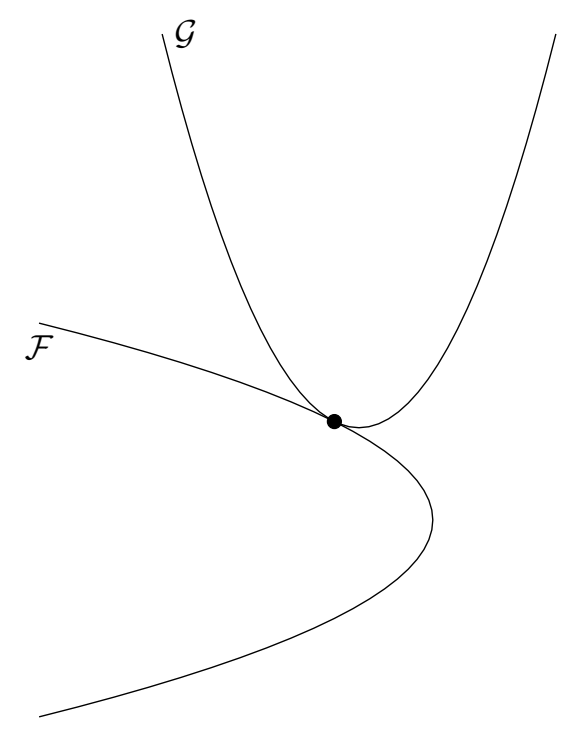

Figure 3.19: If $\mathcal{F}$ and $\mathcal{G}$ are both quadratic, then the only way they can intersect exactly once is for the intersection point to be a tangency.

For cases 5 and 6 , it is clear that there will be exactly one type A fixed point and one type B fixed point. Thus by Theorem 3.4.1 the type A fixed point is not part of a heteroclinic connection, and so we can apply Theorem 3.5.1 to see that $T$ must have a homoclinic point.

For case 7 , one fixed point will be type A, one will be type B, and the other will be a tangency. Thus this case is ruled out by hypothesis.

For case 8 , there wil be two type A fixed points and two type B fixed points. If the type A fixed points do not share a heteroclinic connection then this situation satisfies the hypothesis of Theorem 3.5.1 and we are done. So let us assume that there is a heteroclinic connection between the type A points.

There are four possible orientations for the parabolas in the case of four intersections. All four give rise to one type A1 point and one type A2 point. We can use the results of Section 3.3 to see what the stable and unstable manifolds of these fixed points look like. This is illustrated in Figure 3.20. We see from Figure 3.20 that any heteroclinic connection would have to involve one of the 

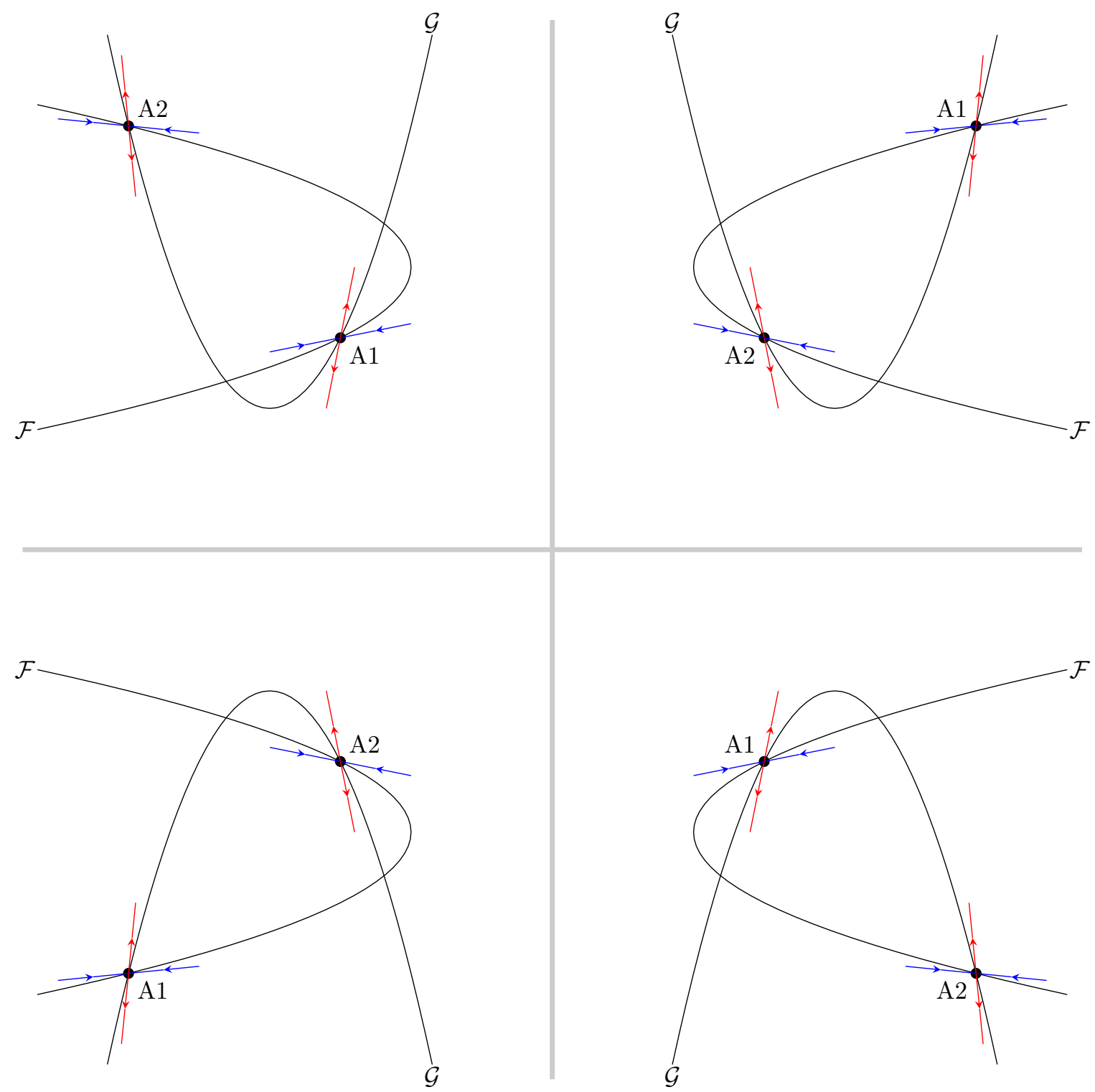

Figure 3.20: Four ways that $\mathcal{F}$ and $\mathcal{G}$ can intersect exactly four times if they are both quadratic. Unstable manifolds are in red and stable manifolds are in blue. 
stable or unstable manifolds crossing either $\mathcal{F}$ or $\mathcal{G}$. By the results of section 2.3, such a crossing must be a homoclinic point since $T$ is both $F$ and $G$ reversible.

So for case 8, the map $T$ must admit a homoclinic point.

This proves the theorem. 


\subsection{Examples}

Now we look at some concrete examples of mappings $T:[f, g]$. Recall that the proof of the Main Theorem was broken down into eight cases, and we proved that cases 5, 6, and 8 admitted a homoclinic point. Case 7, because of its tangential fixed point, did not satisfy the hypothesis of the theorem.

In this section we construct examples of each of cases $5,6,7$, and 8 and use the software package Dynamica [KM2] to visualize the stable and unstable manifolds. In each of the cases, it appears that all homoclinic points are transverse, which indicates the presence of an embedded horseshoe by Smale's theorem (Theorem 2.2.2). 


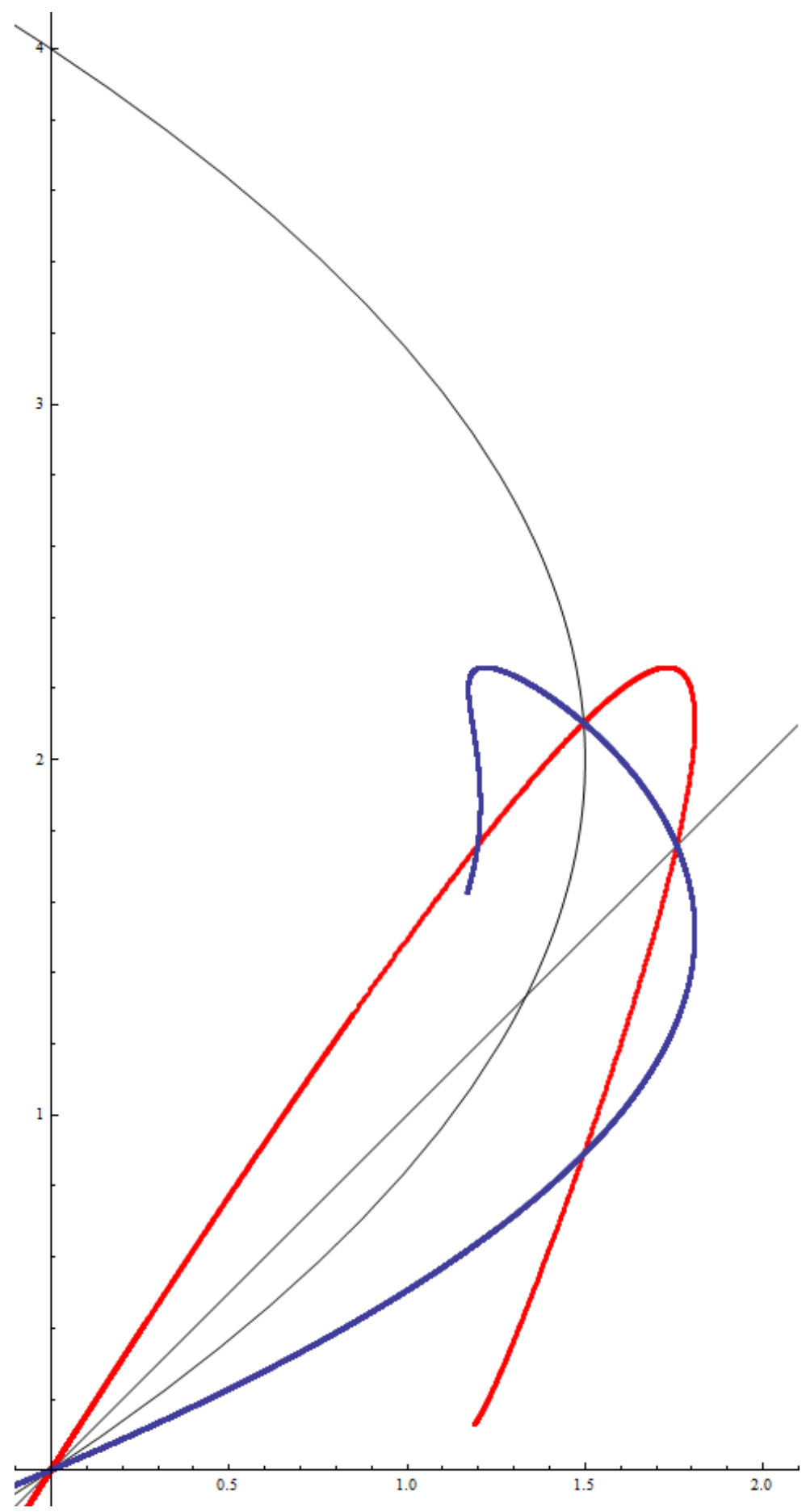

Figure 3.21: Case 5: $T:[f, g]$ with $f(x)=\frac{3}{8} x^{2}-\frac{3}{2} x$ and $g(x)=x$. The blue and red curves are the stable and unstable manifolds, respectively, of the type A1 hyperbolic fixed point at $(0,0)$. The other fixed point is $\left(\frac{4}{3}, \frac{4}{3}\right)$ and is type B elliptic. 


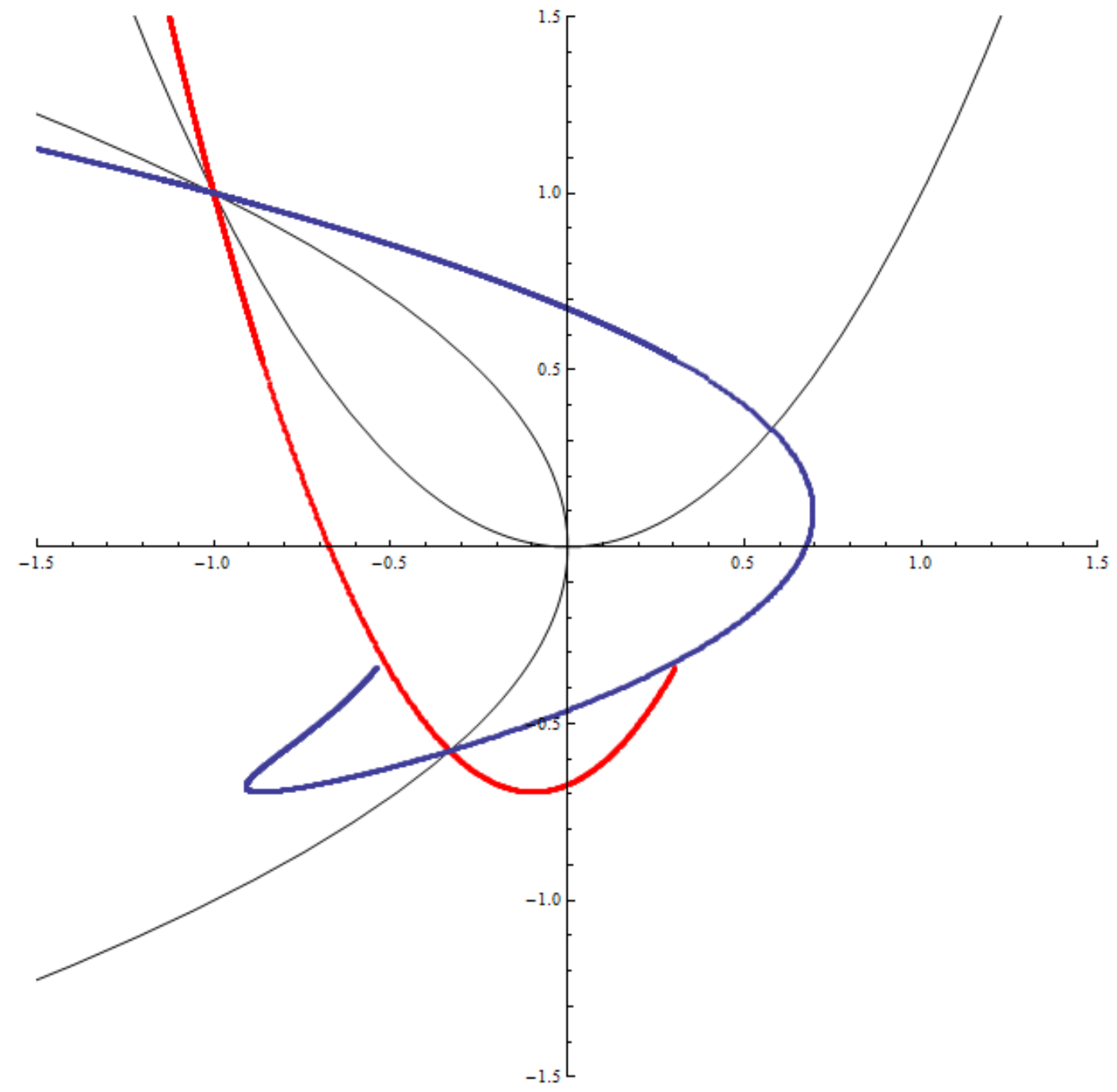

Figure 3.22: Case 6: $f(x)=x^{2}$ and $g(x)=x^{2}$. The blue and red curves are the stable and unstable manifolds, respectively, of the type A2 hyperbolic fixed point at $(-2,2)$. The other fixed point is $(0,0)$ and has a double -1 eigenvalue. 


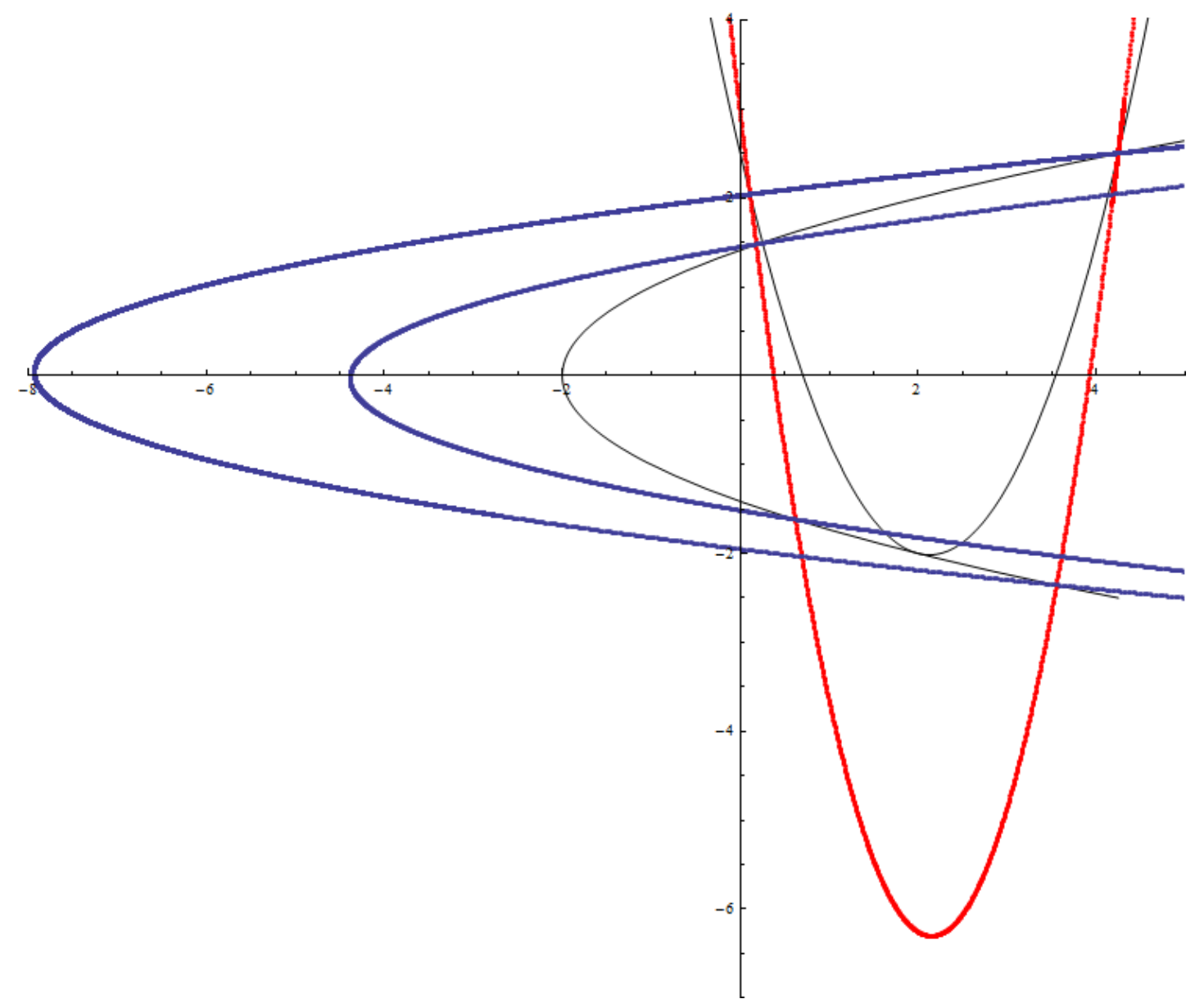

Figure 3.23: Case 7: $f(x)=2-x^{2}$ and $g(x)=x^{2}-4.25 x+2.5$. The blue and red curves are the stable and unstable manifolds, respectively, of the type A1 hyperbolic fixed point at $(4.25,2.5)$. The other fixed points are $(0.25,1.5)$ which is type B2 hyperbolic, and $(2,-2)$ which has a double 1 eigenvalue. 


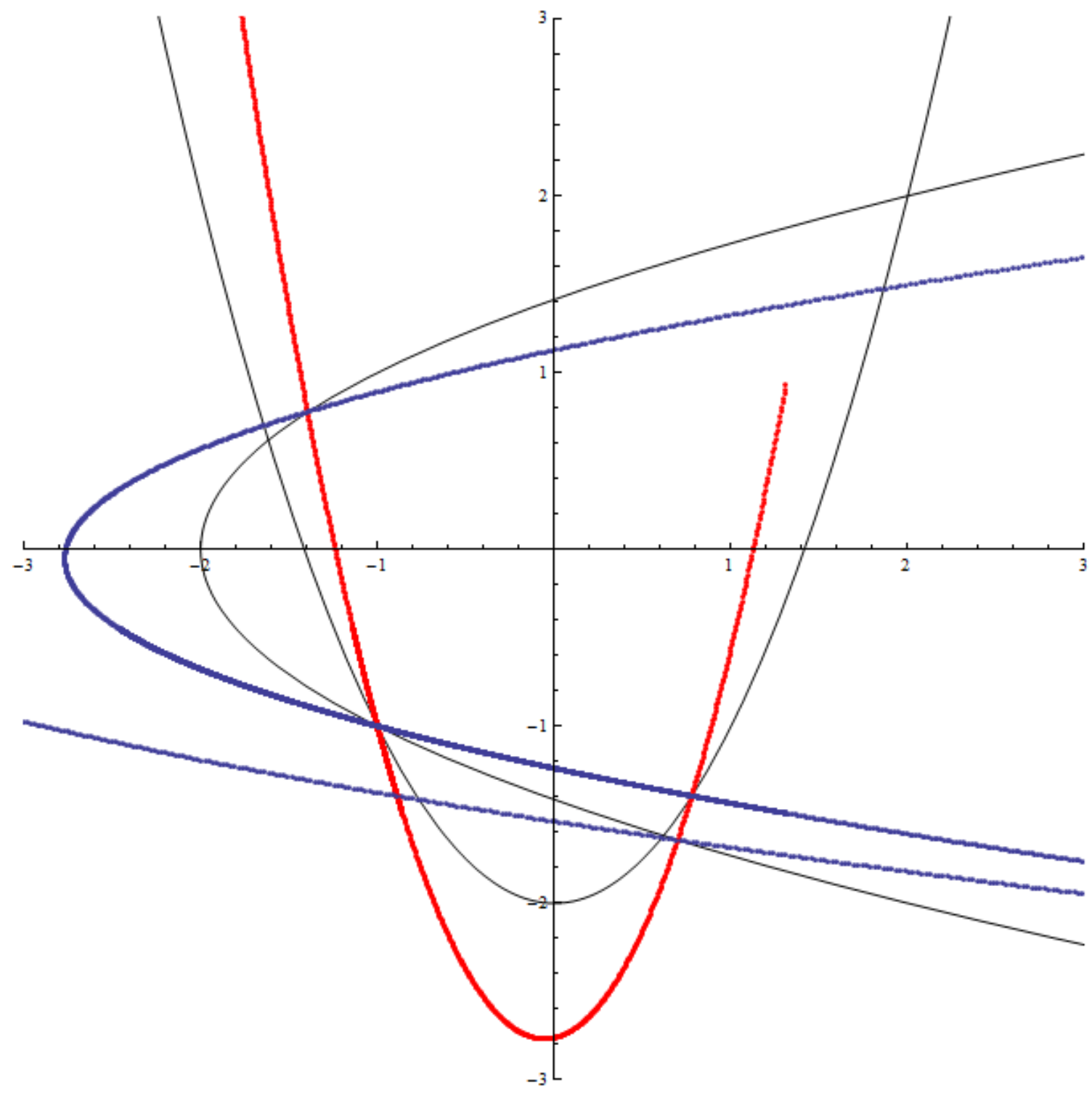

Figure 3.24: Case 8: $f(x)=2-x^{2}$ and $g(x)=x^{2}-2$. The blue and red curves are the stable and unstable manifolds, respectively, of the type A2 hyperbolic fixed point at $(-1,-1)$. The other fixed points are $(2,2)$ which is type A1 hyperbolic, $\left(\frac{-1-\sqrt{5}}{2}, \frac{-1+\sqrt{5}}{2}\right)$ which is type B2 hyperbolic, and $\left(\frac{-1+\sqrt{5}}{2}, \frac{-1-\sqrt{5}}{2}\right)$ which is type B1 hyperbolic. 


\subsection{The Challenge of Proving Conjecture 1.0.1}

In this section we present an example that appears to have a homoclinic loop. Only extremely close inspection reveals that it does not. This illustrates the delicate nature of this conjecture. The angle between $W^{u}$ and $W^{s}$ may be so small that $W^{u}$ and $W^{s}$ appear to coincide! Any method of proof would have to be extremely sensitive to distinguish between this case and the case of an actual homoclinic loop.

We consider the map $T:[f, g]$ with $f(x)=0.59 x(x-2)$ and $g(x)=x$. Again we use the software package Dynamica [KM2] for all computations. T satisfies the hypothesis of the Main Theorem, so it must admit a homoclinic point. $T$ has a type A1 hyperbolic fixed point at $(0,0)$ and a type B elliptic fixed point at $\left(\frac{18}{59}, \frac{18}{59}\right)$. The stable manifold (in blue) and unstable manifold (in red) of the hyperbolic fixed point are shown in Figure 3.25. From this picture, it certainly looks as though there is a homoclinic loop. If we zoom in on the region near the origin, as in Figure 3.26, it still looks like a homoclinic loop. In Figure 3.27 we plot the trajectories of several points inside the homoclinic loop. It seems clear from the diagram that all these trajectories lie on invariant curves, which means that $T:[f, g]$ cannot contain an embedded horseshoe. However, if we zoom in very closely on the homoclinic loop (Figure 3.28 ) we see that that it is in fact not a loop! The stable and unstable manifolds of $(0,0)$ are very close to each other, but do not actually coincide. 


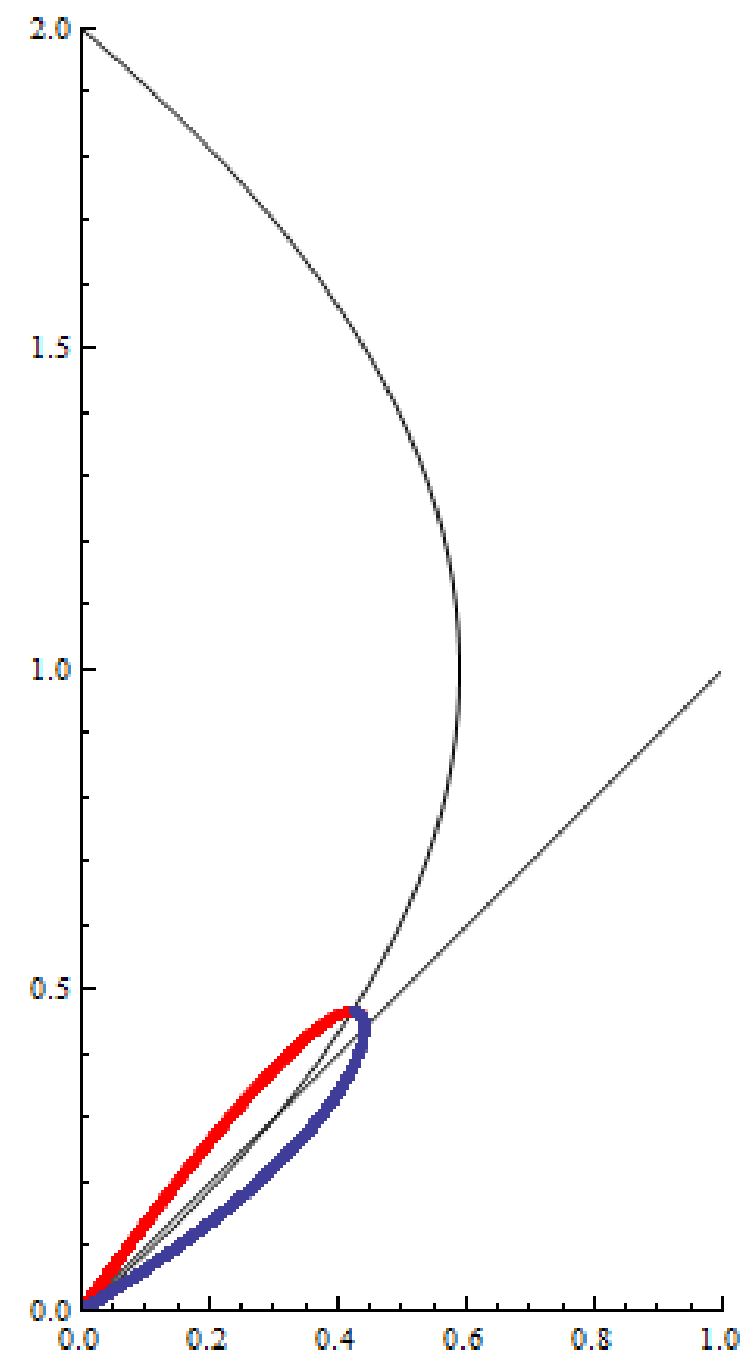

Figure 3.25: $T:[f, g]$ with $f(x)=0.59 x(x-2)$ and $g(x)=x$. The blue and red curves are the stable and unstable manifolds, respectively, of the A1 fixed point at $(0,0)$. 


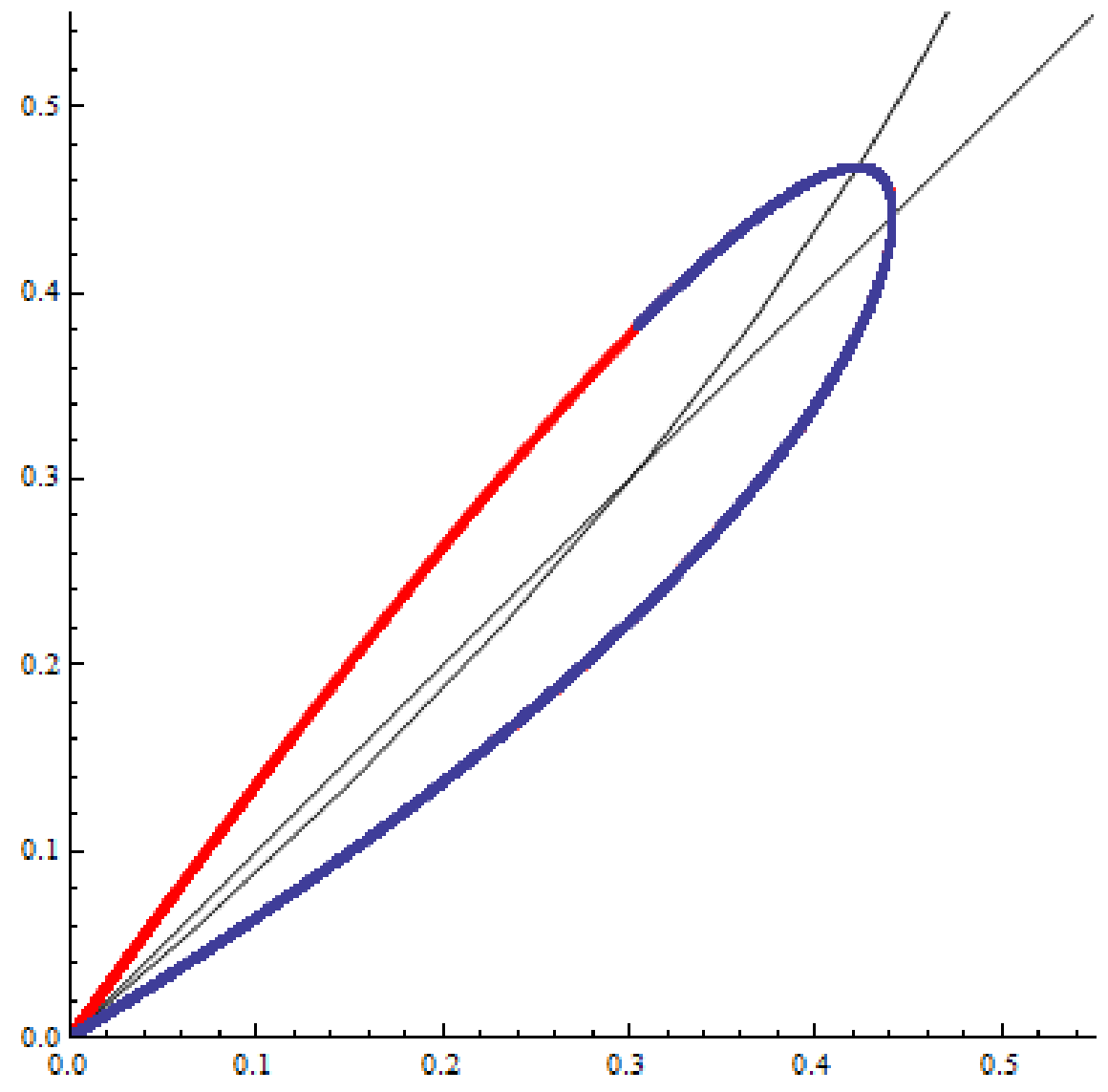

Figure 3.26: Zooming in on the homoclinic loop for $T:[f, g]$ with $f(x)=0.59 x(x-2)$ and $g(x)=x$. The blue and red curves are the stable and unstable manifolds, respectively, of the A1 fixed point at $(0,0)$. 


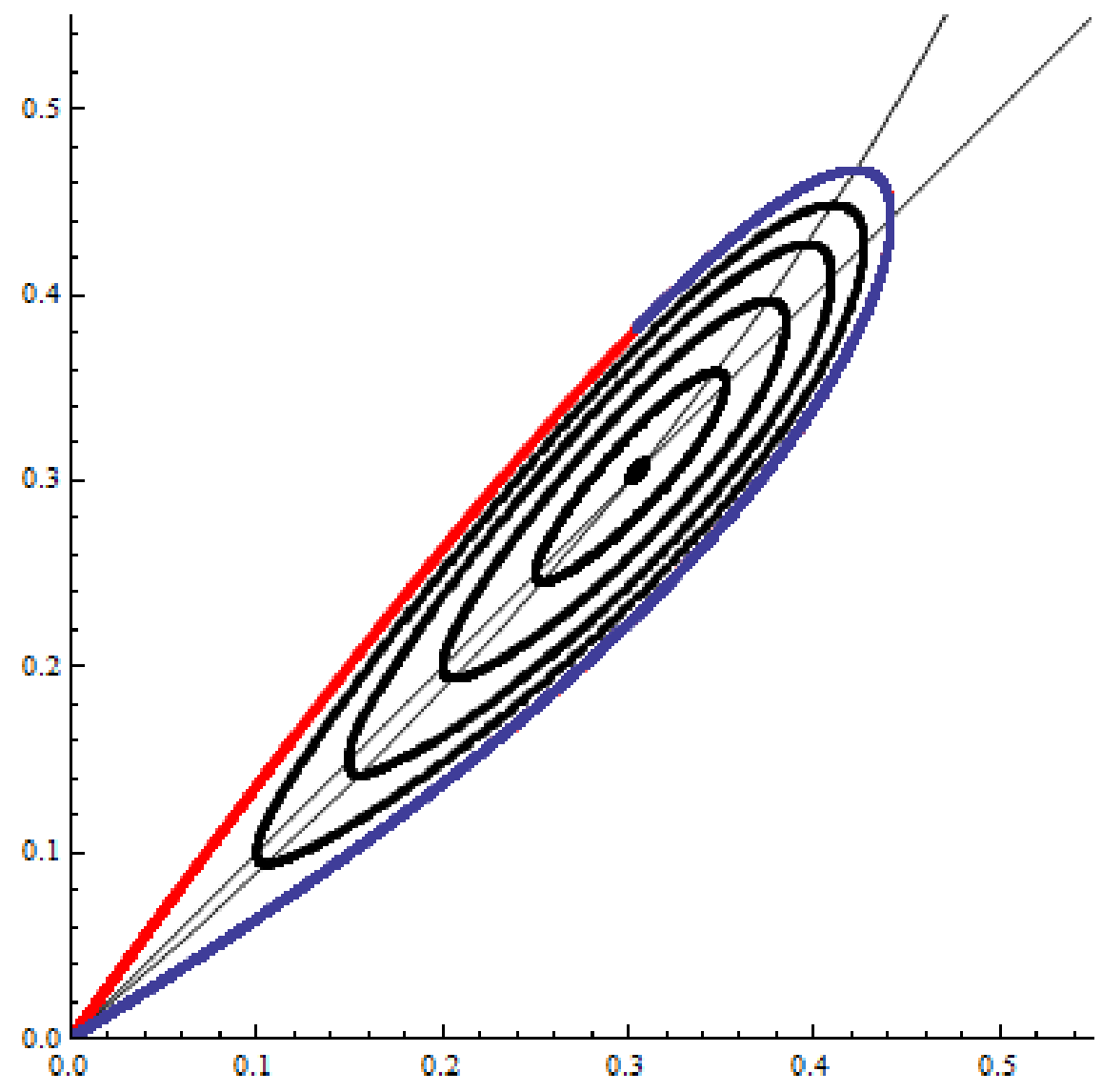

Figure 3.27: $T:[f, g]$ with $f(x)=0.59 x(x-2)$ and $g(x)=x$. The trajectories of points inside the homoclinic loop all seem to lie on invariant curves. 


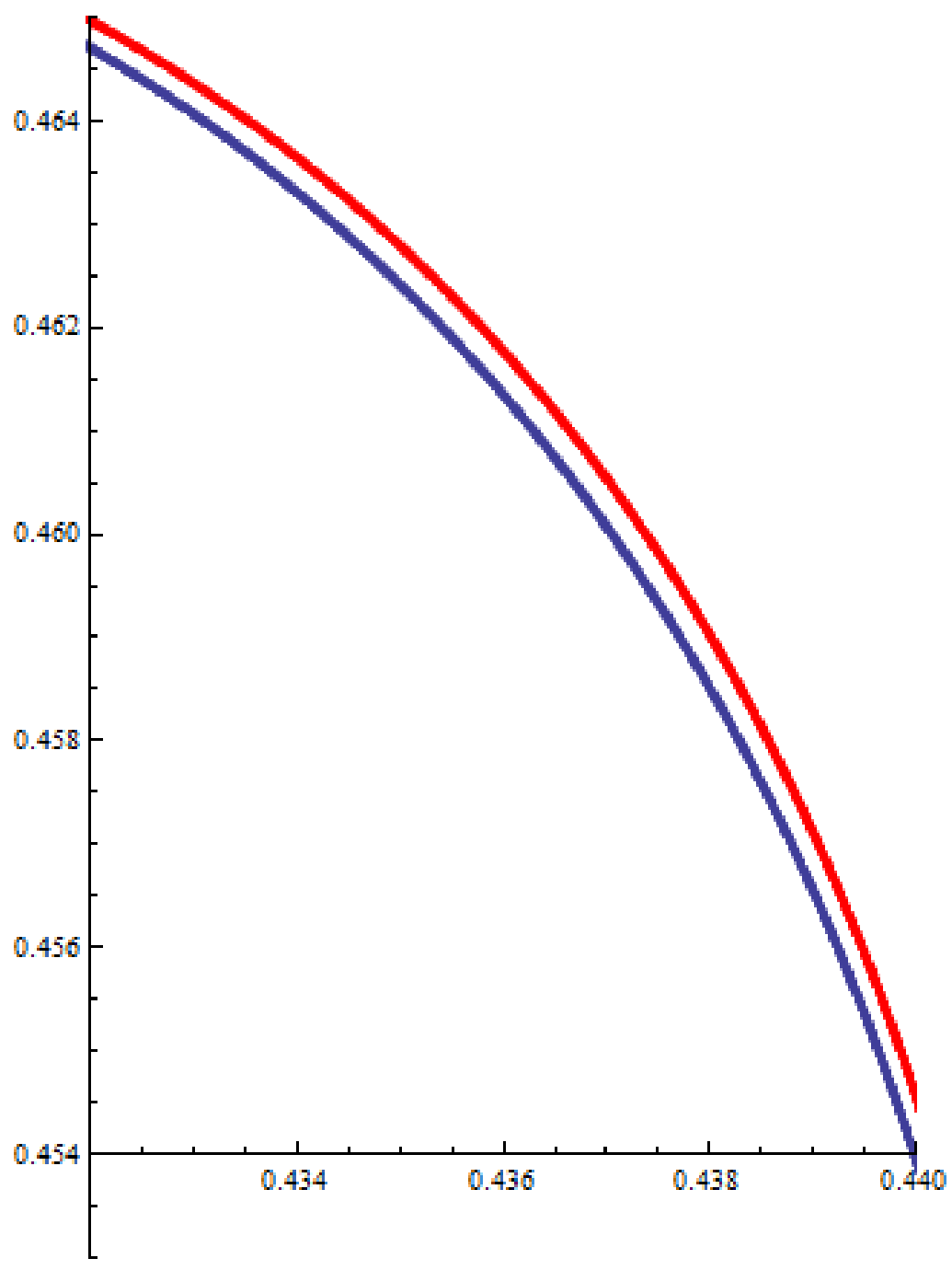

Figure 3.28: An extremely close up view of $W^{u}(0,0)$ (in red) and $W^{s}(0,0)$ (in blue) for $T:[f, g]$ with $f(x)=0.59 x(x-2)$ and $g(x)=x$. 


\subsection{General Conditions that Guarantee the Existence of an Embedded Horseshoe}

In this section we prove the following theorem.

Theorem 3.9.1. Let $f, g: \mathbb{R} \rightarrow \mathbb{R}$ be $C^{1}$ functions with domain all of $\mathbb{R}$, and consider the associated mapping $T:[f, g]$. If the following conditions are satisfied, then $T$ admits an embedded horseshoe.

1. There is at least one type A fixed point that is neither part of a heteroclinic connection nor part of a homoclinic loop.

2. At least one of $f$ or $g$ has either a global maximum or a global minimum.

Just as Theorem 3.5.1 gives general conditions that guarantee the existence of a homoclinic point, Theorem 3.9.1 gives general conditions that guarantee the existence of an embedded horseshoe. We note that if a map $T:[f, g]$ staisfies the hypothesis of Theorem 3.9.1 then it also satisfies the hypothesis of Theorem 3.5.1 and will thus admit a homoclinic point. Our approach to proving Theorem 3.9.1 will be to rule out all the cases where the homoclinic point does not lead to a horseshoe.

If the homoclinic point is transverse, then we can use Smale's theorem (Theorem 2.2.2) to conclude that the map admits an embedded horseshoe.

A natural question to ask is whether or not transversality is a necessary condition. For maps in dimension 2, the answer is no. Consider a map $T: \mathbb{R}^{2} \rightarrow \mathbb{R}^{2}$ with a hyperbolic fixed point $p$, and let $\chi$ be a homoclinic point associated to $p$. As long as the stable and unstable manifolds of $p$ cross at $\chi$ (even if they are tangent while crossing) then the map $T$ will contain an embedded horseshoe. There are two key results which lead to this conclusion, and both of them involve the concept of topological entropy. Informally, the topological entropy of a map $T$, denoted $h(T)$, is a measure of the complexity of the orbit structure of $T$. If $h(T)>0$, then the map may be thought of as 
chaotic. For example, the horseshoe map has positive topological entropy. The first of the key results is due to Katok. In 1980, he published a paper 'Lyapunov exponents, entropy, and periodic orbits for diffeomorphisms' $[\mathrm{K}]$, in which he proved that a map with positive topological entropy must contain an embedded horseshoe. However, this result is only valid in dimension 2 . The second key result is due to Burns and Weiss. In their 1995 paper 'A geometric criterion for positive topological entropy' $[\mathrm{BW}]$, they proved that a map $T: \mathbb{R}^{2} \rightarrow \mathbb{R}^{2}$ has positive topological entropy if the stable and unstable manifolds of a hyperbolic fixed point cross topologically. So, combining these results, we can see that transversality is not a necessary condition. As long as the stable and unstable manifolds of a hyperbolic fixed point cross, then the map $T: \mathbb{R}^{2} \rightarrow \mathbb{R}^{2}$ will contain an embedded horseshoe.

We state this as a theorem.

Theorem 3.9.2. Let $H: \mathbb{R}^{2} \rightarrow \mathbb{R}^{2}$ be a $C^{1}$ diffeomorphism with a hyperbolic fixed point $p$. If $p$ admits a homoclinic point with a topological crossing, then $H$ contains an embedded horseshoe.

So, if the homoclinic point is a tangential crossing (illustrated in Figure 3.29) then the map will contain an embedded horseshoe.

The only other possibilities for the homoclinic point are a non-crossing tangency and a homoclinic loop. The following lemma rules out a non-crossing tangency.

Lemma 3.9.1. Consider a map $T:[f, g]$ that satisfies the hypothesis of Theorem 3.9.1. Let $p$ be a type A fixed point that is neither part of a heteroclinic connection nor part of a homoclinic loop, and let $\chi$ be the associated homoclinic point that Theorem 3.5 .1 guarantees to exist. Let $W^{u}(p)$ and $W^{s}(p)$ be tangent at $\chi$. Then $\chi$ must be a tangential crossing.

Proof. We will consider the case where $\chi$ is the first intersection of $W^{u}(p)$ with $\mathcal{F}$ (as in Figure 3.29). The case where $\chi$ is the first intersection of $W^{u}(p)$ with $\mathcal{G}$ is completely analogous.

The key fact that makes this proof work is that $W^{u}(p)$ is a reflection in $\mathcal{F}$ of $W^{s}(p)$, as a consequence of the map $T$ being $F$ reversible (see Section 2.3). This fact implies that $W^{u}(p)$ and 


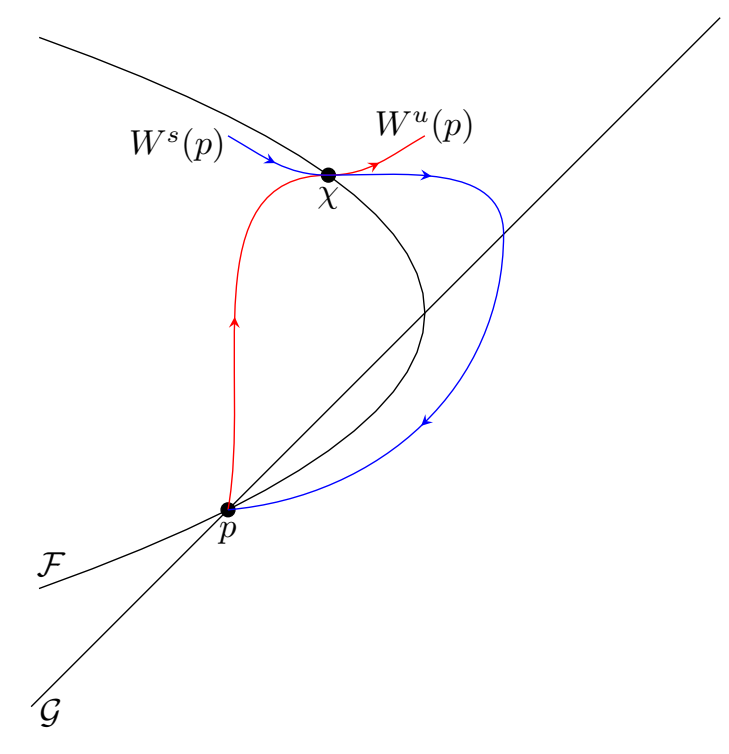

Figure 3.29: A tangential crossing at the homoclinic point $\chi$.

$W^{s}(p)$ must have slope 0 when they intersect $\mathcal{F}$ at $\chi$. The segment of $W^{u}(p)$ from $p$ to $\chi$ together with the segment of $W^{s}(p)$ from $p$ to $\chi$ form a closed curve $C$. By hypothesis, $C$ cannot be a homoclinic loop. Then there are only two possibilities for $W^{u}(p)$ to continue after $\chi \cdot W^{u}(p)$ can go inside $C$ or outside $C$. If it goes outside $C$, then so must $W^{s}(p)$, since $W^{s}(p)$ is a reflection in $\mathcal{F}$ of $W^{u}(p)$. If $W^{u}(p)$ goes inside $C$, then for the same reason so must $W^{s}(p)$. In either case, we have that $\chi$ is a tangential crossing.

This proves the lemma.

So, ruling out the possibility of a homoclinic loop proves the existence of a horseshoe.

Now we may prove Theorem 3.9.1.

Proof of Theorem 3.9.1. By Theorem 3.5.1, we know that $T:[f, g]$ admits a homoclinic point $\chi$. There are four possibilities for $\chi$.

1. $\chi$ is transverse. 
2. $\chi$ is a tangential crossing.

3. $\chi$ is a non-crossing tangency.

4. $\chi$ is part of a homoclinic loop.

Case 1 implies the existence of a horseshoe by Smale's theorem (Theorem 2.2.2). Case 2 implies the existence of a horseshoe by Theorem 3.9.2. Case 3 is impossible by Lemma 3.9.1. So, if case 4 does not occur then $T:[f, g]$ must contain an embedded horseshoe. 


\section{Chapter 4}

\section{Further Research}

\subsection{Arbitrary Angle $\alpha$}

In this thesis, we have studied maps of the form $T:[f, g, \alpha]$ where $\alpha=\frac{\pi}{2}$. We have suppressed the dependence on $\alpha$ in the notation and have denoted the map $T:\left[f, g, \frac{\pi}{2}\right]$ by $T:[f, g]$. In this section, we give a precise description of the maps $T:[f, g, \alpha]$ for arbitrary $\alpha$, and state a conjecture that is analogous to the Main Theorem.

We make the following definitions.

Let $f, g: \mathbb{R} \rightarrow \mathbb{R}$ be $C^{1}$ functions with domain all of $\mathbb{R}$.

Let $\tilde{F}: \mathbb{R}^{2} \rightarrow \mathbb{R}^{2}$ denote a vertical reflection in the graph of $y=f(x)$.

Let $G: \mathbb{R}^{2} \rightarrow \mathbb{R}^{2}$ denote a vertical reflection in the graph of $y=g(x)$.

Let $\alpha \in(0, \pi)$ be an angle.

Let $R_{\alpha}: \mathbb{R}^{2} \rightarrow \mathbb{R}^{2}$ denote a counterclockwise rotation by $\alpha$ about the origin.

Let $R_{-\alpha}: \mathbb{R}^{2} \rightarrow \mathbb{R}^{2}$ denote a clockwise rotation by $\alpha$ about the origin.

Let $F=R_{\alpha} \circ \tilde{F} \circ R_{-\alpha}$. 
The expressions $\tilde{F}, G, R_{\alpha}, R_{-\alpha}$, and $F$ are given by

$$
\begin{aligned}
\tilde{F}\left(\begin{array}{l}
x \\
y
\end{array}\right) & =\left(\begin{array}{c}
x \\
2 f(x)-y
\end{array}\right) \\
G\left(\begin{array}{l}
x \\
y
\end{array}\right) & =\left(\begin{array}{c}
x \\
2 g(x)-y
\end{array}\right) \\
R_{\alpha}\left(\begin{array}{l}
x \\
y
\end{array}\right) & =\left(\begin{array}{l}
x \cos \alpha-y \sin \alpha \\
x \sin \alpha+y \cos \alpha
\end{array}\right) \\
R_{-\alpha}\left(\begin{array}{l}
x \\
y
\end{array}\right) & =\left(\begin{array}{c}
x \cos \alpha+y \sin \alpha \\
-x \sin \alpha+y \cos \alpha
\end{array}\right) \\
F\left(\begin{array}{l}
x \\
y
\end{array}\right) & =\left(\begin{array}{l}
x \cos 2 \alpha+y \sin 2 \alpha-2 f(x \cos \alpha+y \sin \alpha) \sin \alpha \\
x \sin 2 \alpha-y \cos 2 \alpha+2 f(x \cos \alpha+y \sin \alpha) \cos \alpha
\end{array}\right)
\end{aligned}
$$

So the maps we consider are of the form $T=G \circ F$ and are given by

$T\left(\begin{array}{l}x \\ y\end{array}\right)=\left(\begin{array}{c}x \cos 2 \alpha+y \sin 2 \alpha-2 f(x \cos \alpha+y \sin \alpha) \sin \alpha \\ 2 g(x \cos 2 \alpha+y \sin 2 \alpha-2 f(x \cos \alpha+y \sin \alpha) \sin \alpha)-x \sin 2 \alpha+y \cos 2 \alpha-2 f(x \cos \alpha+y \sin \alpha) \cos \alpha\end{array}\right)$

This leads us to make the following definition.

Definition 4.1.1. Let $f, g: \mathbb{R} \rightarrow \mathbb{R}$ be $C^{1}$ functions with domain all of $\mathbb{R}$, and let $\alpha \in(0, \pi)$ be an angle. We denote the mapping $T$ associated with the triple $(f, g, \alpha)$ by $T:(f, g, \alpha)$ and define $T$ by $T\left(\begin{array}{l}x \\ y\end{array}\right)=\left(\begin{array}{c}x \cos 2 \alpha+y \sin 2 \alpha-2 f(x \cos \alpha+y \sin \alpha) \sin \alpha \\ 2 g(x \cos 2 \alpha+y \sin 2 \alpha-2 f(x \cos \alpha+y \sin \alpha) \sin \alpha)-x \sin 2 \alpha+y \cos 2 \alpha-2 f(x \cos \alpha+y \sin \alpha) \cos \alpha\end{array}\right)$

Let $\mathcal{F}$ denote the fixed point set of $F$ and let $\mathcal{G}$ denote the fixed point set of $G$. The set $\mathcal{F}$ will be the graph of the function $y=f(x)$ rotated by $\alpha$ counterclockwise. The set $\mathcal{G}$ will be the graph of the function $y=g(x)$. 
Geometrically, $F$ is a reflection in the curve $\mathcal{F}$ along the vector $(-\sin \alpha, \cos \alpha), G$ is a vertical reflection in the curve $\mathcal{G}$, and $T:(f, g, \alpha)$ is the composition of the two reflections. For example, let $f(x)=(x+1)^{2}, g(x)=1$ and $\alpha=\frac{\pi}{4}$. Figure 4.1 illustrates the effect of $T:[f, g, \alpha]$ on a point $v$.

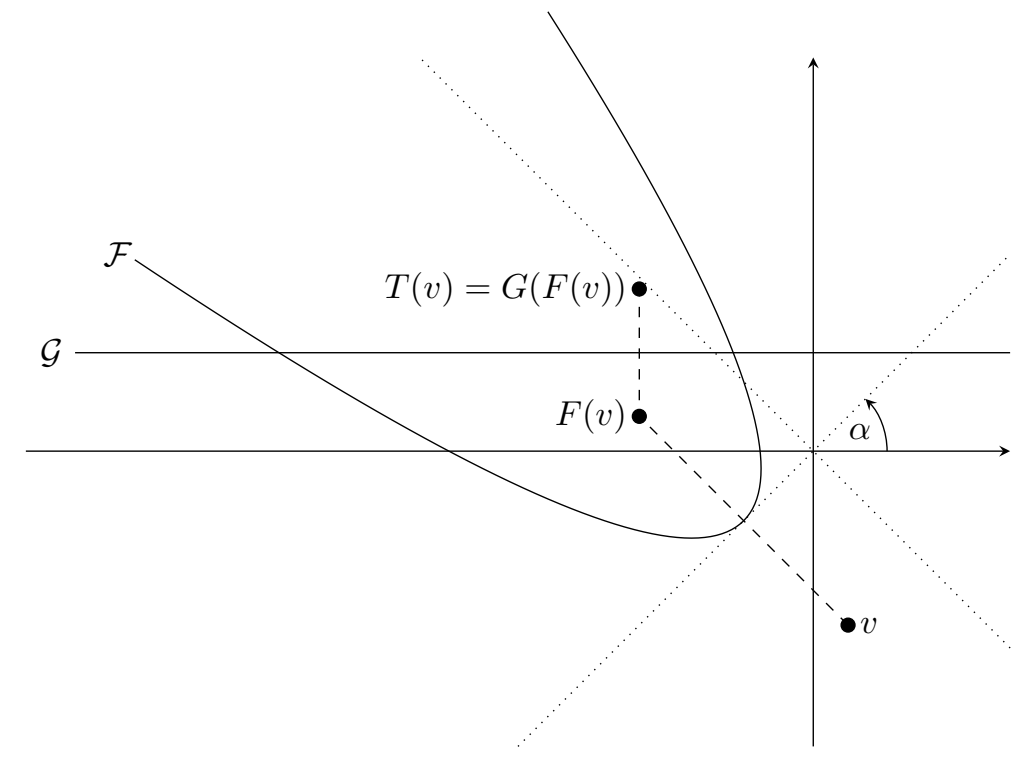

Figure 4.1: The effect of $T:[f, g, \alpha]$ for $f(x)=(x+1)^{2}, g(x)=1$, and $\alpha=\frac{\pi}{4}$.

It is easy to see from the definition of $T:[f, g, \alpha]$ how restricting $\alpha$ to $\frac{\pi}{2}$ simplifies calculations. However, it seems to me that the essential geometry of the situation remains the same for any angle $\alpha$. I believe that that the following modified version of the Main Theorem will still be true.

Conjecture 4.1.1. Let $f, g: \mathbb{R} \rightarrow \mathbb{R}$ be $C^{1}$ functions with domain all of $\mathbb{R}$, let $\alpha \in(0, \pi)$ be an angle, and consider the associated mapping $T:[f, g, \alpha]$. Let $\mathcal{F}$ denote the curve $\left(\begin{array}{l}x \\ y\end{array}\right)=\left(\begin{array}{c}\cos \alpha \\ \sin \alpha\end{array}\right) t+\left(\begin{array}{c}-\sin \alpha \\ \cos \alpha\end{array}\right) f(t)$, and let $\mathcal{G}$ denote the graph of $y=g(x)$. Then the following two statements are true.

1. If one of $f$ or $g$ is quadratic and the other is linear, then the map $T$ will admit a homoclinic point if and only if $\mathcal{F}$ and $\mathcal{G}$ intersect more than once.

2. If $f$ and $g$ are both quadratic, then the map $T$ will admit a homoclinic point if and only if $\mathcal{F}$ 
and $\mathcal{G}$ intersect more than once.

As a piece of evidence for this conjecture, consider the area preserving Hénon map with parameter $a \neq 0$, given by

$$
H\left(\begin{array}{l}
x \\
y
\end{array}\right)=\left(\begin{array}{c}
1+y-a x^{2} \\
-x
\end{array}\right) .
$$

$H$ is conjugate to $T:[f, g, \alpha]$ for $f(x)=0, g(x)=\frac{1}{2}\left(a x^{2}-1\right)$ and $\alpha=\frac{\pi}{4}$. For $a>-1$, we have that the curves $\mathcal{F}$ and $\mathcal{G}$ will intersect twice, and that $H$ admits a homoclinic point [D1]. In fact, Brown has proved that $H$ will admit an embedded horseshoe for $a>0[\mathrm{Br}]$.

I think that the most elegant way to prove Conjecture 4.1.1 would be to find a conjugacy with the case proved in the Main Theorem. For example, suppose $T:[f, g, \alpha]$ satisfies the hypothesis of Conjecture 4.1.1. One would first find functions $f_{1}$ and $g_{1}$ such that $T:[f, g, \alpha]$ is conjugate to $T_{1}:\left[f_{1}, g_{1}, \frac{\pi}{2}\right]$. Then one would verify that $T_{1}:\left[f_{1}, g_{1}, \frac{\pi}{2}\right]$ satisfies the hypothesis of the Main Theorem, and thus contains a homoclinic point. Then, since $T$ and $T_{1}$ are conjugate, the map $T:[f, g, \alpha]$ must also contain a homoclinic point. I am not sure, however, if finding an appropriate conjugacy will always be possible.

Another way to approach proving Conjecture 4.1.1 would be to use a proof of the Main Theorem as a template. I think that this approach would work, although the technical difficulties of dealing with an arbitrary angle $\alpha$ would be significant. 


\section{Bibliography}

[B-G] June Barrow-Green, Poincaré and the Three Body Problem, American Mathematical Society, Providence, RI, 1997.

[Bo] R. Bowen, Topological entropy and axiom A, Global Analysis, Proc. Sympos. Pure Math., Amer. Math. Soc. 14 (1970), 23-42.

[Br] Ray Brown, Horseshoes in the measure preserving Hénon map, Ergod. Th. and Dyn. Sys. 15 (1995), 1045-1059.

[BW] K. Burns and H. Weiss, A geometric criterion for positive topological entropy, Comm. Math. Phys. 192 (1995), 95-118.

[CFL1] X. Cabré, E. Fontich, and R. de la Llave, The parameterization method for invariant manifolds I: Manifolds associated to non-resonant subspaces, Indiana Univ. Math. J. 52 (2003), 283-328.

[CFL2] X. Cabré, E. Fontich, and R. de la Llave, The parameterization method for invariant manifolds II: Regularity with repect to parameters, Indiana Univ. Math. J. 52 (2003), $328-360$.

[CFL3] X. Cabré, E. Fontich, and R. de la Llave, The parameterization method for invariant manifolds III: Overview and applications, J. Differential Equ. 218 (2005), 444-515. 
[CFN1] J. Casasayas, E. Fontich, and A. Nunes, Invariant manifolds for a class of parabolic points, Nonlinearity 5 (1992), 1193-1210.

[CFN2] J. Casasayas, E. Fontich, and A. Nunes, Homoclinic orbits to parabolic points, Nonlinear Differential Equ. Appl. 4 (1997), 201-216.

[CR] R. Churchill and D. Rod, Pathology in dynamical systems III: Analytic Hamiltonians, J. Differential Equ. 37 (1980), 23-38.

[Co] C. Conley, in 'Topological Dynamics', Joseph Auslander, ed., pp 129-153, Benjamin, New York, 1968.

[DN] R. Devaney and Z. Nitecki, Shift automorphisms in the Hénon mapping, Comm. Math. Phys. 67 (1979), 137-148.

[D1] R. Devaney, Homoclinic bifurcations and the area-conserving Hénon mapping, J. Differential Equ. 51 (1984), 254-266.

[D2] R. Devaney, An Introduction to Chaotic Dynamical Systems, Westview Press, Colorado, 1989.

[F] E. Fontich, Transversal homoclinic points of a class of conservative diffeomorphisms, J. Differential Equ. 87 (1990), 1-27.

[GS1] N. Gavrilov and L. Silnikov, Three-dimensional dynamical systems that are close to systems with a structurally unstable homoclinic curve I, Mat. Sb. (N.S.) 88(130) (1972), 475-492.

[GS2] N. Gavrilov and L. Silnikov, Three-dimensional dynamical systems that are close to systems with a structurally unstable homoclinic curve II, Mat. Sb. (N.S.) 90(132) (1973), 139-156.

[GL] R. Gilmore and M. Lefranc, The Topology of Chaos, John Wiley \& Sons, Inc., New York, 2002. 
[Ha] W. Haidegger, Melnikov's vector - a "measure of chaos", Rep. Math. Phys. 30 (1991), $223-231$.

[H1] M. Hénon, A two dimensional mapping with a strange attractor, Comm. Math. Phys. 50 (1976), 65-80.

[HPS] M. Hirsch, C. Pugh, and M. Shub, Invariant Manifolds, Springer-Verlag, New York, 1977.

[H2] M. Hénon, Numerical study of quadratic area-preserving mappings, Quart. Appl. Math. XXVII(3) (1969), 291-312.

[Ho] D. Hobson, An efficient method for computing invariant manifolds of planar maps, J. Comp. Phys. 104 (1993), 14-22.

[HOV] A. Homburg, H. M. Osinga, and G. Vegter, On the computation of invariant manifolds for fixed points, Z. Angew. Math. Phys. 46 (1995), 171-187.

[HW] A. Homburg and H. Weiss, A geometric criterion for positive topological entropy II: Homoclinic tengencies, Comm. Math. Phys. 208 (1999), 267-273.

[J1] E. Jensen, Horseshoes in the measure preserving Hénon map for all even exponents. M.Sc. thesis, Queen's University, 2004.

[J2] E. Jensen, A new construction of the unstable manifold for the measure-preserving Hénon map, Proc. Amer. Math. Soc. 136 (2008), 181-192.

[K] A. Katok, Lyapunov exponents, entropy and periodic orbits for diffeomorphisms, Publ. Inst. Hautes Etudes Sci. 51 (1980), 137-173.

[KY] J. Kennedy and J. Yorke, Toplogical horseshoes, Trans. Amer. Math. Soc. 353 (2001), $2513-2530$.

[Kn] J. Knobloch, Chaotic behaviour near non-transversal homoclinic points with quadratic tangency, J. Difference Equ. Appl. 12 (2006), 1037-1056. 
[KYY1] E. Kostelich, J. Yorke, and Z. You, Plotting stable manifolds: error estimates and noninvertible maps, Phys. D 93 (1996), 210-222.

[KYY2] E. Kostelich, J. Yorke, and Z. You, Calculating stable and unstable manifolds, Internat. J. Bifur. Chaos 1 (1991), 605-623.

[KM1] M. R. S. Kulenovic and O. Merino, Discrete Dynamical Systems and Difference Equations with Mathematica, Chapman \& Hall/CRC, Boca Raton, 2002.

[KM2] M. R. S. Kulenovic and O. Merino, Dynamica: A computer package for the study of Discrete Dynamical Systems and Difference Equations. http://www.math.uri.edu/Dynamica/

[L] E. N. Lorenz, Deterministic non-periodic flow, J. Atmospheric Sci. 20 (1963), 130-141.

[M1] F. Marotto, Snap-back repellers imply chaos in $\mathbb{R}^{n}$, J. Math. Anal. Appl. 63 (1978), $199-223$.

[M2] F. Marotto, Chaotic behaviour in the Hénon mapping, Comm. Math. Phys. 68 (1979), $187-194$.

[M3] F. Marotto, On redefining a snap-back repeller, Chaos Solitons Fractals 25 (2005), 25-28.

[MM] R. McGehee and K. Meyer, Homoclinic points of area preserving diffeomorphisms, Amer. J. Math. 96 (1974), 409-421.

[NR] A. Neumaier and T. Rage, Rigorous chaos verification in discrete dynamical systems, Phys. D. 67 (1993), 327-346.

[Ra] V. Rayskin, Homoclinic Tangencies in $\mathbb{R}^{n}$, Discrete and Continuous Dyn. Sys. 12 (2005), 465-480.

[Ro] C. Robinson, Dynamical Systems, CRC Press, Florida, 1995. 
[S1] S. Smale, Diffeomorphisms with many periodic points. In 'Differential and Combinatorial Topology' pp. 63-80, Princeton University Press, Princeton, New Jersey, 1965.

[S2] S. Smale, Finding a horseshoe on the beaches of Rio, Math. Intelligencer 20(1) (1998), $39-44$.

[T] M. Tabor, Chaos and Integrability in Nonlinear Dynamics, Wiley-Interscience, New York, 1989.

[W] P. Walters, Introduction to Ergodic Theory, Springer-Verlag, New York, 1982.

[Y] X-S. Yang, Topological horseshoes and computer assisted verification of chaotic dynamics, Internat. J. Bifur. Chaos Appl. Sci. Engrg. 19 (2009), 1127-1145. 